\title{
Evaluation of Individual Finger Forces During Activities of Daily Living In Healthy Individuals and Those with Hand Arthritis
}

\author{
Michael Riddle, The University of Western Ontario \\ Supervisor: Lalone, Emily A., The University of Western Ontario \\ Co-Supervisor: Ferreira, Louis M., The University of Western Ontario \\ A thesis submitted in partial fulfillment of the requirements for the Master of Engineering \\ Science degree in Biomedical Engineering \\ (C) Michael Riddle 2019
}

Follow this and additional works at: https://ir.lib.uwo.ca/etd

Part of the Biomedical Devices and Instrumentation Commons

\begin{abstract}
Recommended Citation
Riddle, Michael, "Evaluation of Individual Finger Forces During Activities of Daily Living In Healthy Individuals and Those with Hand Arthritis" (2019). Electronic Thesis and Dissertation Repository. 6247. https://ir.lib.uwo.ca/etd/6247
\end{abstract}

This Dissertation/Thesis is brought to you for free and open access by Scholarship@Western. It has been accepted for inclusion in Electronic Thesis and Dissertation Repository by an authorized administrator of Scholarship@Western. For more information, please contact wlswadmin@uwo.ca. 


\begin{abstract}
Hand-Osteoarthritis (H-OA) leads to pain, loss of grip strength, and decreased hand function. Current treatment for H-OA involves joint protection programs (JPP) which seek to reduce joint loading during activity. The use of wearable technology to measure hand forces during activity has the potential to determine the effectiveness of JPP. The objective of this thesis was to develop and validate a method of directly measuring finger forces during the performance of activities of daily living, and then use that system to measure the envelope of hand forces during activity in healthy individuals and in patients with $\mathrm{H}-\mathrm{OA}$. A commercially-available capacitive sensor system was validated for use in this application and found an envelope of applied forces consistent with previous literature. Using the measurement system and protocols presented in this thesis, the effectiveness of JPP at reducing hand forces can, for the first time, be objectively quantified.

\section{Keywords}

Hand Osteoarthritis (H-OA); Joint Protection Programs (JPP); Activities of Daily Living (ADL); Hand Function; Hand Forces; Wearable Technology; Force Sensors 


\section{Summary for Lay Audience}

Joint protection programs (JPP) help patients with hand arthritis reduce the forces in their hands to reduce pain and improve function. These programs focus on training people to use alternate movements, devices, and strategies that can reduce forces on their hands in daily tasks. While these programs have been designed using basic principles, we know very little about the actual forces applied by the hands during home and work tasks. Without this knowledge, we do not know how to best reduce joint loads and minimize pain. This thesis explores the use of small sensors which slide over the fingers to measure these finger forces during the performance of activities. Both healthy people and those with hand arthritis were recruited to participate in this study and the range of forces applied by the fingers during several activities was measured with these sensors. This represents the first step in evaluating current joint protection programs to determine if they result in lower forces applied by the fingers. 


\section{Co-Authorship Statement}

Chapter 1: Michael Riddle - sole author

Chapter 2: Michael Riddle - concept and design of study, data collection, interpretation of data, drafting document; Sydney Robinson - data collection; Joy MacDermid - concept and design of study, reviewed manuscript; Mike Szekeres - interpretation of data, reviewed manuscript; Louis Ferreira - concept and design of study, interpretation of data, reviewed manuscript; Emily Lalone - concept and design of study, interpretation of data, reviewed manuscript.

Chapter 3: $\quad$ Michael Riddle - sole author 


\section{Table of Contents}

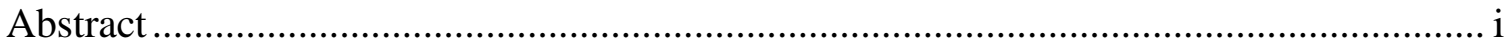

Co-Authorship Statement..................................................................................

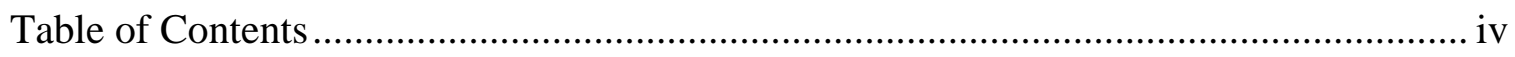

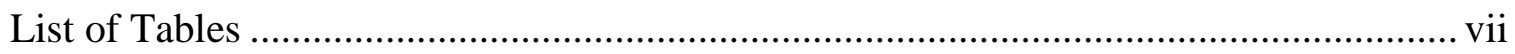

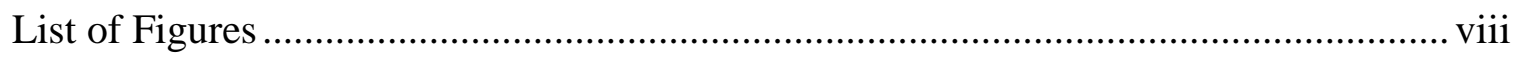

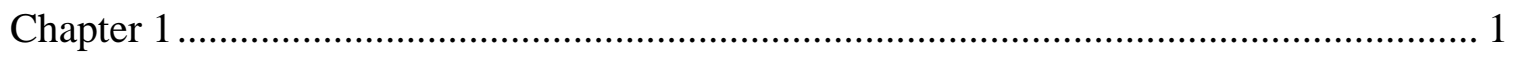

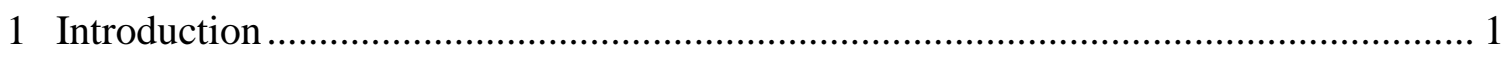

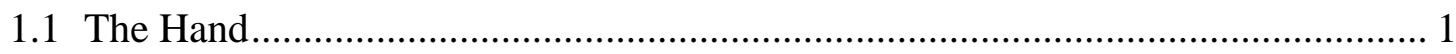

1.1.1 Anatomy and Range of Motion......................................................... 1

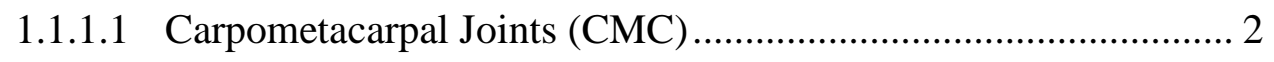

1.1.1.2 Metacarpophalangeal Joints (MCP) ...................................... 2

1.1.1.3 Interphalangeal Joints (IP) ............................................. 3

1.1.2 Musculature / Force Generation........................................................... 3

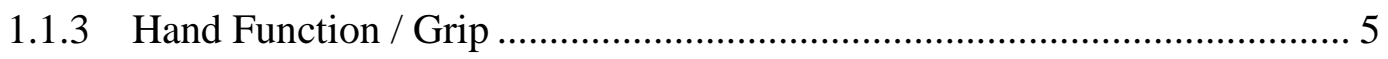

1.2 Hand Osteoarthritis .............................................................................. 7

1.2.1 Effect on Hand Function ............................................................... 7

1.3 Biomechanical Models of the Hand.......................................................... 8

1.4 Current Methods of Hand Force Measurement ........................................... 9

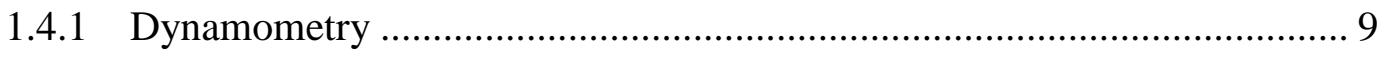

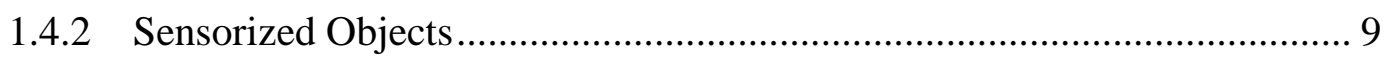

1.4.3 Force Gloves / Sensors Attached to the Hand ....................................... 9

1.4.4 Strain Gauge and Capacitive Sensors ................................................ 10

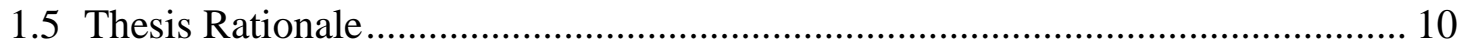

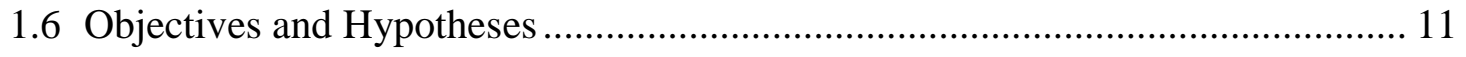




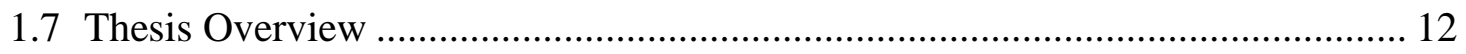

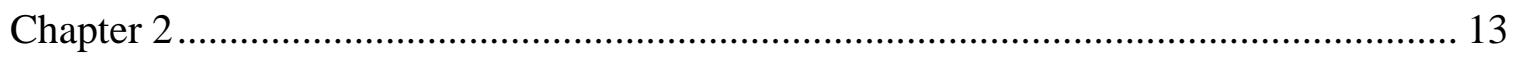

2 Individual Finger Forces during Activities of Daily Living ……................................ 13

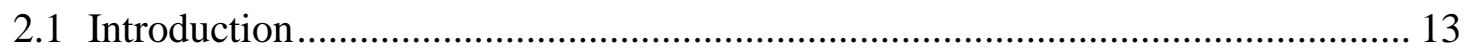

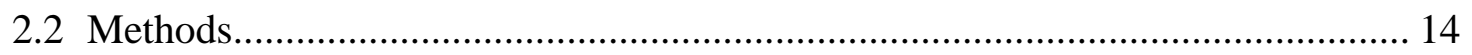

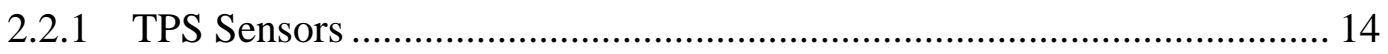

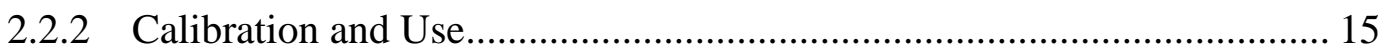

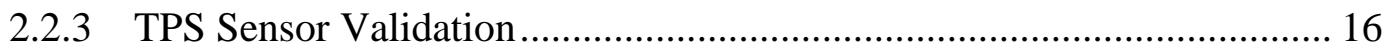

2.2.3.1 Load Cell Comparison.............................................................. 16

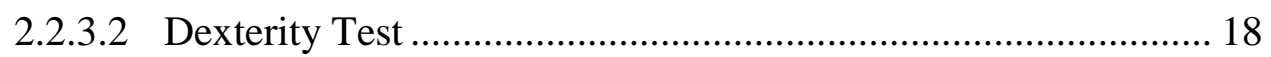

2.2.3.3 Data Acquisition and Analysis .................................................. 19

2.2.4 Hand Force Measurement During ADL ……......................................... 20

2.2.4.1 Study Protocol ......................................................................... 20

2.2.4.2 Data Acquisition and Analysis ................................................. 21

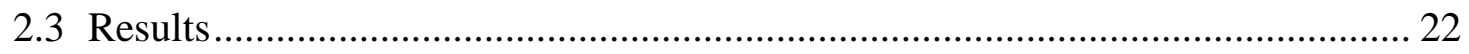

2.3.1 FingerTPS Sensor Validation ............................................................. 22

2.3.1.1 Load Cell Comparison................................................................. 22

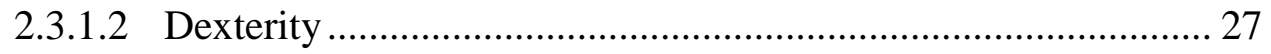

2.3.2 Forces during Daily Tasks ................................................................... 29

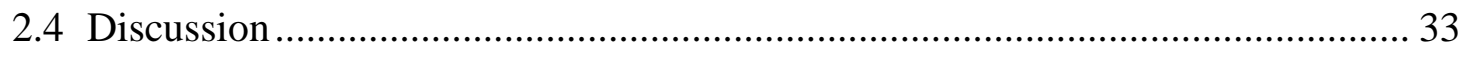

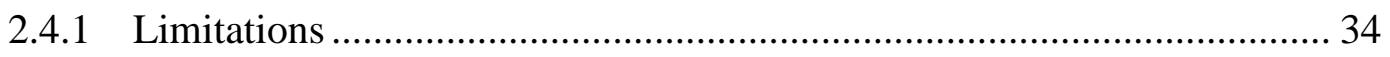

2.4.2 Future Work ……………………………….................................. 35

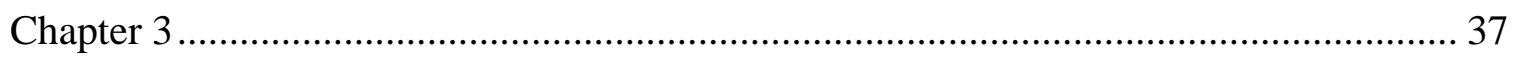

3 General Discussion and Conclusion ................................................................... 37

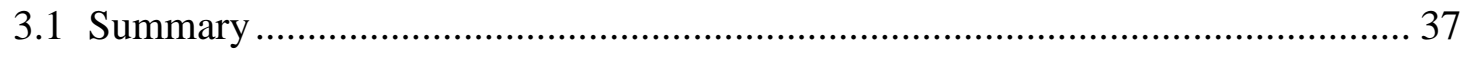

3.2 Significance 


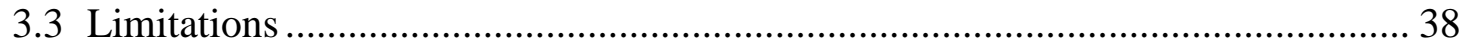

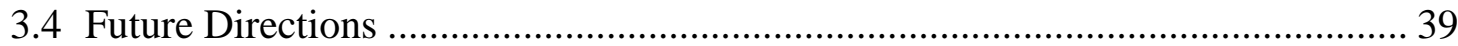

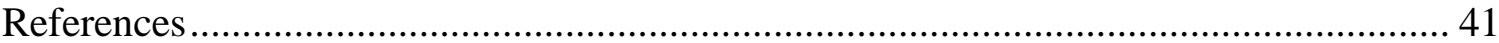

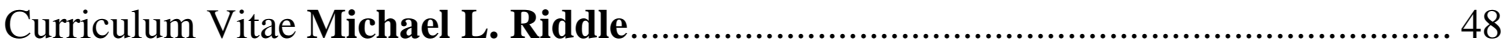




\section{List of Tables}

Table 2.1. The tasks from this study were selected from those commonly found on three psychometric evaluations: Patient Rated Wrist and Hand Evaluation (PRWHE), Disabilities of the Arm, Shoulder, and Hand (DASH), and Joint Protection Behavior Assessment (JPBA). Other ubiquitous tasks were added to our evaluation to get a better picture of the envelope of forces. * indicates task which were listed generically on the evaluation (turning on a tap and

turning a doorknob) but were further specified for this study (lever vs. standard). 21 


\section{List of Figures}

Figure 1.1 Bones and Joints of the Hand. 2

Figure 1.2. Major muscles, tendons, and ligaments involved in finger flexion and extension. 4

Figure 1.3. Collateral ligaments in the MCP, PIP, and DIP joints which allow for motion in extension (top) and flexion (bottom) of the fingers 5

Figure 1.4 - Common Precision and Power Grips ......................................................... 7

Figure 2.1 - Capacitive sensors are located at the fingertips (volar pad) of the thumb and first three fingers. 14

Figure 2.2 - Complete FingerTPS setup with four sensors (one each on the thumb and first three fingers), wrist module, and Bluetooth transmitter. 15

Figure 2.3 - Calibration of FingerTPS sensors using provided load cell. 16

Figure 2.4 Shear force measurement setup.

Figure 2.5. The N.K. Dexterity Board was used to assess the dexterity of individuals with bare hands and while wearing the FingerTPS sensors

Figure 2.6 Scatterplot and Bland-Altman plots comparing the measurement of force from the FingerTPS sensors and Loadcell. 23

Figure 2.7. Frequency Plot showing the distribution of variation in recorded force value between FingerTPS and load cell. $(n=141)$.

Figure 2.8. Boxplots indicating the spread of the differences at three time points to examine drift over time. 25

Figure 2.9 Example force over time for the loadcell in the $\mathrm{X}, \mathrm{Y}$, and $\mathrm{Z}$ directions and the FingerTPS sensors when lateral (x-direction) shear is applied by the index finger.

Figure 2.10 Force over time for the loadcell in the X, Y, and Z directions and the FingerTPS sensors when longitudinal (y-direction) shear is applied by the index finger. 27 
Figure 2.11. Average time and standard deviation to complete the dexterity board with bare hands and while wearing the TPS sensors.

Figure 2.12. Max force graphed for each finger in Newtons with standard error for tasks dominated by the thumb and index finger (precision grip).

Figure 2.13. Max force graphed for each finger in Newtons with standard error for tasks that rely on all four fingers (thumb, index, middle, ring) to generate force (power grip). 31

Figure 2.14. Max force graphed for each finger in Newtons with standard error for tasks with some other combination of primary movers (power and precision grips) 32 


\section{Chapter 1}

\section{Introduction}

This chapter reviews the relevant anatomy of the hand which allows for the generation for force required to interact with the world around us, and how impairment to the hand in the form of injury or disease impede our ability to perform functional tasks. This chapter also examines the deficiencies of current biomechanical models and methods of measuring finger forces. The chapter concludes with the rationale and objectives of this research as well as an overview of the following chapters.

\subsection{The Hand}

The human hand is our primary tool used for mechanical interactions with the environment around us [1]. Previous studies have estimated that during a typical eight hour work day (for a housemaid and a machinist), an average worker performs between 4000 and 5000 grip changes [2]. Our hands are complex end effectors made up of many bones, muscles, tendons, and ligaments allowing for over 20 degrees of freedom which enable us to perform very precise movements and also exert high forces [3]. As a result of the kinematic structure of the upper extremity, our hands and fingers have a high degree of dexterity and are capable of performing a variety of fine motor movements which allow us to perform activities of daily living (ADL) [4], [5].

\subsubsection{Anatomy and Range of Motion}

The hand and wrist consist of 27 bones -8 carpal bones, 5 metacarpal bones, and 14 phalanges. The joints of the hand involved in hand movement, beginning with the joints at the connection between the metacarpals and distal row of carpal bones and moving distally to the tips of the phalanges, will be described below. All the major joints and bones of the hand involved in articulation of the fingers are labeled in Figure 1.1. 


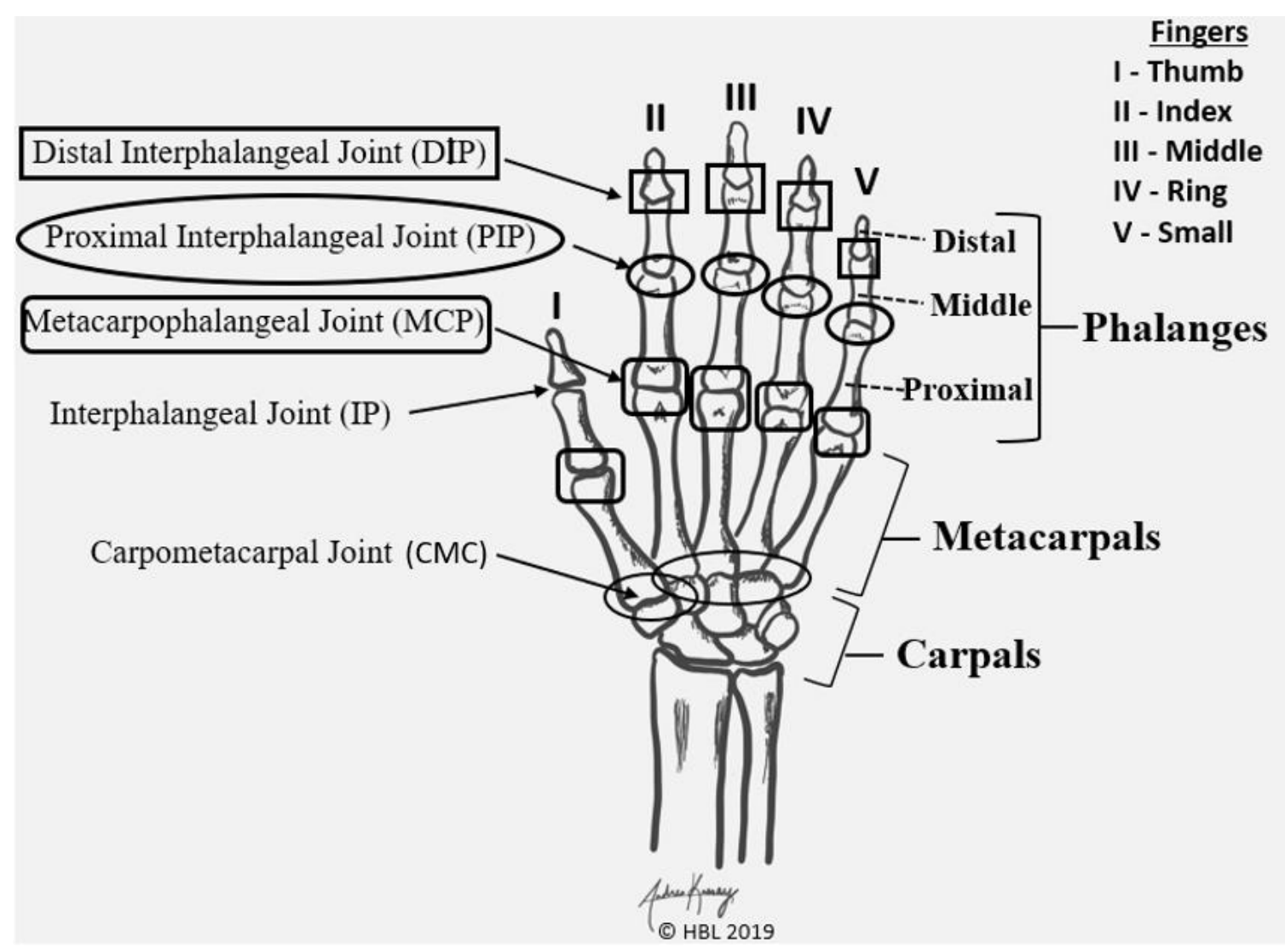

Figure 1.1 Bones and Joints of the Hand.

\subsubsection{Carpometacarpal Joints (CMC)}

The CMC joints are located where the distal row of carpals connect to the four fingers and the thumb by the metacarpals. In the four fingers, the CMC joint is a gliding joint which allows for very little motion in flexion and extension [6]. However, in the thumb the CMC is a saddle joint between the trapezium and the first metacarpal which allows for most of the thumb's articulation [6]. The thumb CMC allows for 10 to $15^{\circ}$ of rotation, 40 to $80^{\circ}$ of abduction and adduction, as well as 50 to $80^{\circ}$ of flexion and extension [6]. The large range of motion allowed by the thumb CMC in humans greatly increases the function of the hand.

\subsubsection{Metacarpophalangeal Joints (MCP)}

The next joint, moving distally down the hand, is the union of the metacarpals and proximal phalanges at the MCP joints. In the four fingers, the MCP joint is a condyloid joint allowing for motion in both flexion-extension as well as abduction-adduction. Finger flexion is 
between 70 and $90^{\circ}$ and is least in the index finger and greatest in the little (small) finger [7]. Finger extension at the $\mathrm{MCP}$ is roughly $25^{\circ}$ and is limited by the position of the wrist [6]. In adduction and abduction, the fingers are able to move approximately $20^{\circ}$ [8]. As with the $\mathrm{CMC}$, the function of this joint differs between the thumb and the four fingers. In the thumb the MCP is a hinge joint which only allows motion in one plane, flexion and extension. In flexion the thumb can move between 30 and $90^{\circ}$, and in extension, $15^{\circ}$ [8].

\subsubsection{Interphalangeal Joints (IP)}

The most distal joints in the hand are the interphalangeal (IP) joints which connect the phalanges in the fingers and thumb: 3 phalanges in the four fingers (proximal, middle, and distal) and only 2 in the thumb (proximal and distal). Each of the four fingers, then, has two IP joints: the proximal interphalangeal (PIP) and distal interphalangeal (DIP) joints; whereas, the thumb only has one IP joint. The IP joints are hinge joints allowing for motion in one plane only, flexion and extension [6]. The range of motion allowed by these joints is $110^{\circ}$ at the PIP joints and $90^{\circ}$ at the DIP of the four fingers and IP of the thumb [6].

\subsubsection{Musculature / Force Generation}

The force generated by the hand would not be possible without the complex musculature acting at the hand and wrist and collateral ligaments that restrict motion to certain directions. Most of the muscles acting in the wrist and finger joints are extrinsic muscles which originate outside of the hand near the elbow and enter the hand as tendons, some of which are quite long and terminate on the tip of the distal phalanges [6]. Other, intrinsic, muscles originate in the hand to initiate motion of the MCP and IP joints [6]. The major muscles, tendons, and ligaments involved in hand motion are labeled in Figures 1.2 and 1.3. 


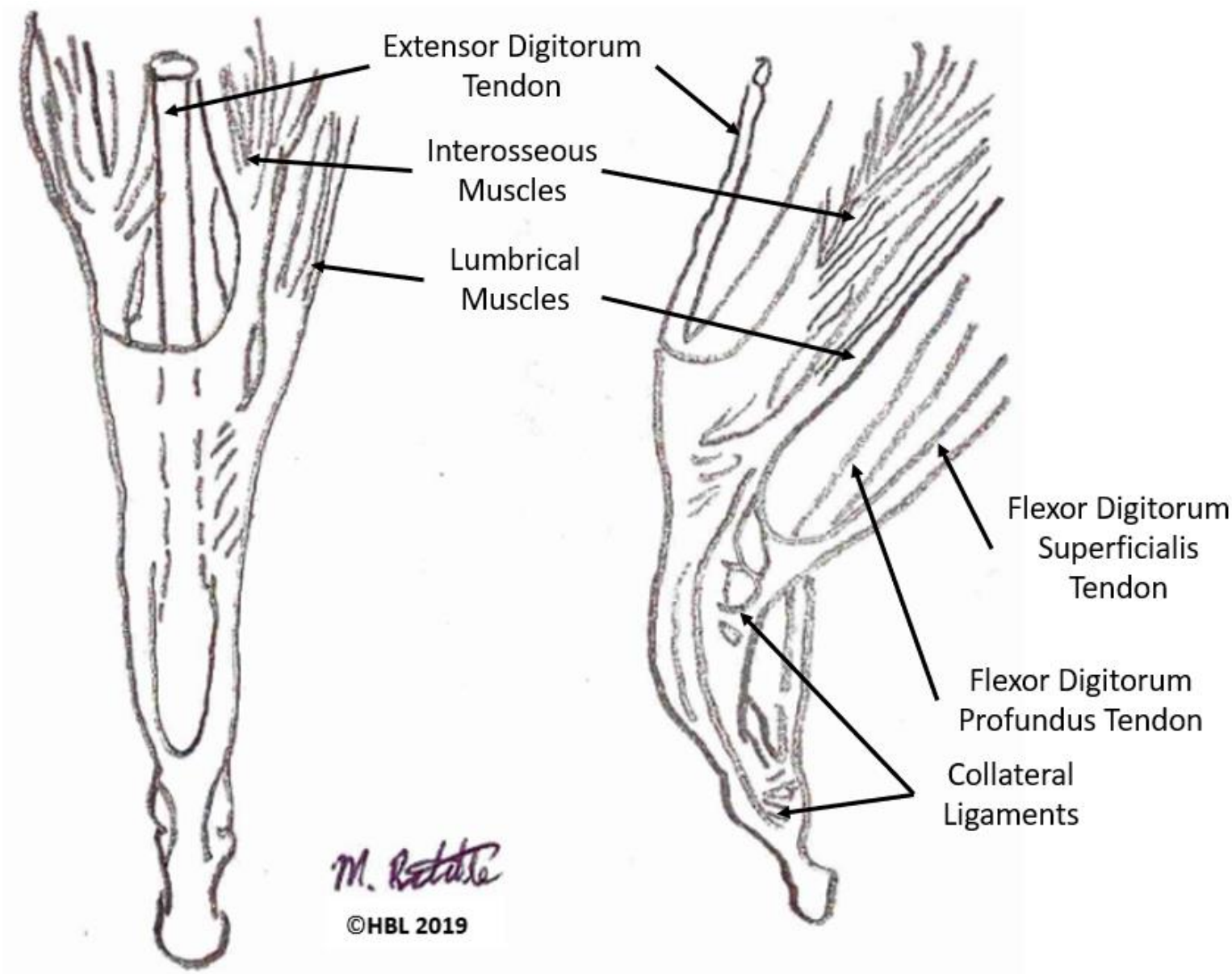

Figure 1.2. Major muscles, tendons, and ligaments involved in finger flexion and extension. 


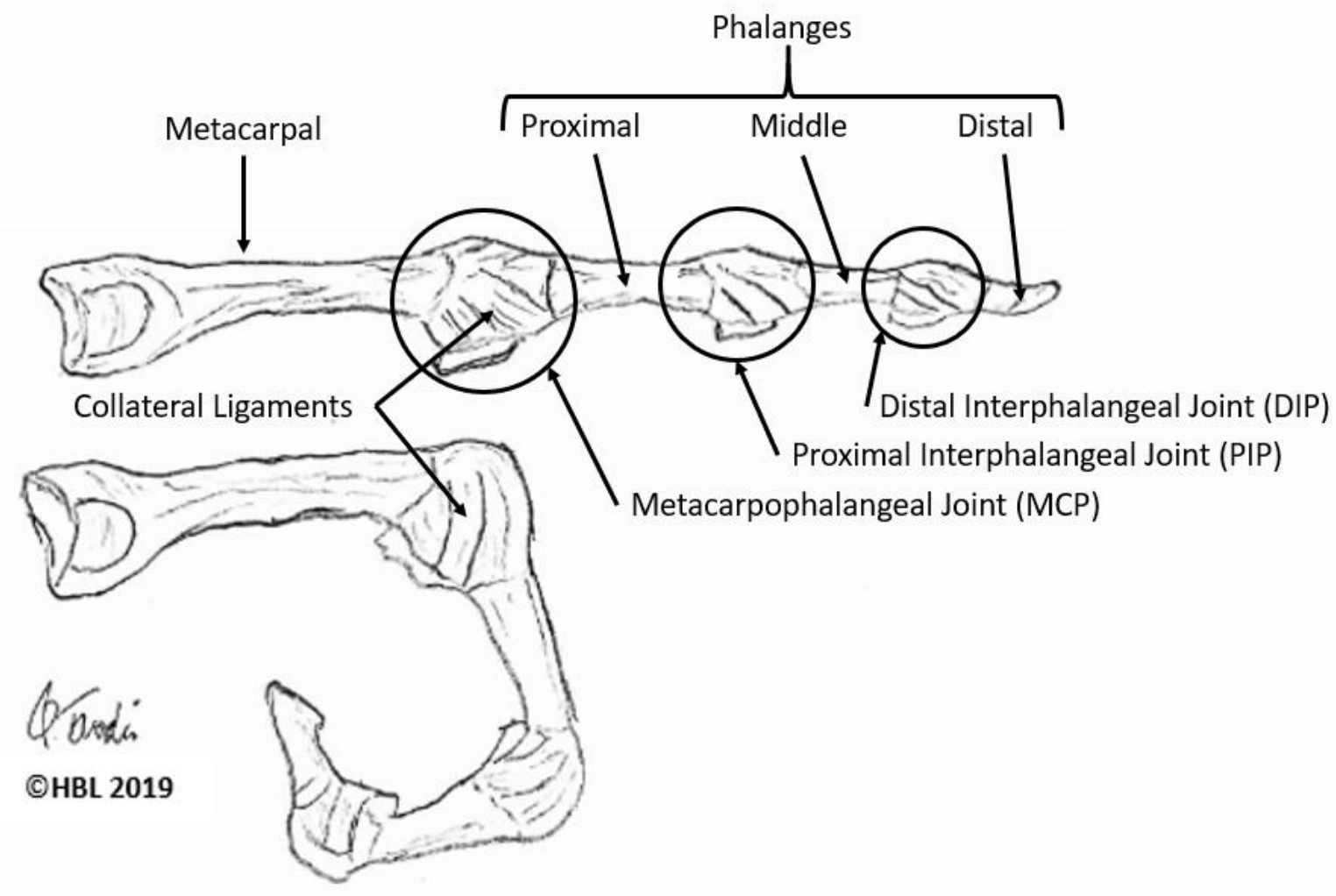

Figure 1.3. Collateral ligaments in the MCP, PIP, and DIP joints which allow for motion in extension (top) and flexion (bottom) of the fingers.

Flexion of the fingers at the IP joints is primarily controlled by the flexor digitorum profundus and the flexor digitorum superficialis [9]. These are extrinsic muscles that originate in the forearm and enter the hand as flexor tendons. Flexion at the MCP joints is produced by two intrinsic sets of muscles, the lumbricales and the interossei [6]. For extension of the fingers, the primary muscle is the extensor digitorum which originates in the forearm and enters the hand as four tendon slips which branch off at the MCP [9]. The lumbricales and interossei also play a role in the extension of the PIP and DIP joints [6]. When gripping objects, the extrinsic muscles are the primary movers when the grip requires maximum output, whereas the intrinsic muscles are primarily for more precise gripping such as pinching [6].

\subsubsection{Hand Function / Grip}

Normal hand function is essential to be able to interact with the world around us. We use our hands to perform nearly every activity from dressing and grooming to vocational and recreational activities [10]. In an average workday alone, it has been reported that we 
perform between 4000 and 5000 grip changes [2]. However, the most common activities are classed as activities of daily living (ADLs), and represent the routine tasks people perform daily to participate in daily life [10]. These activities are often the focus of clinical evaluation of normal hand function [2]. Common methods of determining normal hand function involve the assessment an individual's grip strength and dexterity, the ability to manipulate the hands to perform tasks. Dexterity is commonly assessed clinically by observing the time it takes for individuals to manipulate various sized objects with the hands [11], [12].

Grip strength is determined, primarily, by the flexion strength at the MCP and IP joints in the fingers [6], [13]. Grip is generally classed into one of two categories: power grip and precision grip [14]. For power grip, there is more flexion of the fingers and for maximum output there is flexion at all three finger joints, MCP, PIP, and DIP [6], [13]. In this grip, the thumb remains in the plane of the hand and typically either wraps around the object or the fully flexed fingers [6]. For precision grip, there is typically limited flexion at the PIP and DIP joints ant only one or two fingers is involved [6]. In this grip the thumb is typically perpendicular to the hand. Examples of power and precision grip are shown in Figure 1.4. 


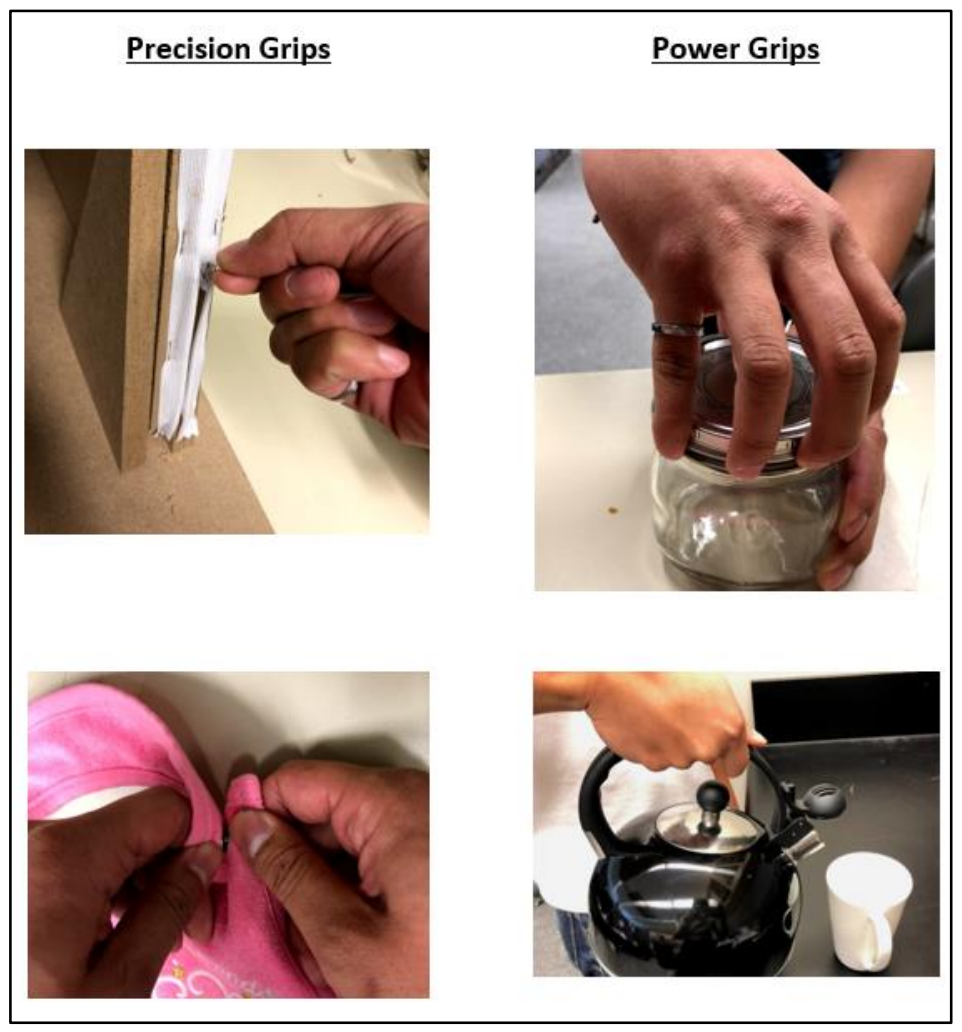

Figure 1.4 - Common Precision and Power Grips

\subsection{Hand Osteoarthritis}

Hand-osteoarthritis (H-OA) is one of the leading causes of decreased hand function in adults and is the most common disease of the hand affecting over $20 \%$ of the population [5], [15]. Osteoarthritis is generally described as the degradation of articular cartilage resulting from genetic factors, wear and tear of the joint, or a post-traumatic response to injury [16]. H-OA is defined as osteoarthritis which affects the CMC, PIP and DIP joints of the hand and is most prevalent in the older population and in women [17]. Pain and stiffness of the joints are among the most common symptoms of H-OA [18].

\subsubsection{Effect on Hand Function}

Impairment to the fingers as a result of trauma, autoimmune diseases, and degenerative diseases greatly impede our ability to perform functional tasks [5]. In addition to a reduction in dexterity of the fingers, these impairments often result in pain whenever force is applied to the hands. Because of decreased grip strength or pain when loading the joint, a major 
issue that is associated with $\mathrm{H}-\mathrm{OA}$ is the decrease in hand function. Individuals with osteoarthritis are often unable to complete activities of daily living or must make modifications to the way they perform these activities to compensate for their pain and decreased function [15]. Both grip strength and range of motion are significantly impaired in individuals with $\mathrm{H}-\mathrm{OA}$ [19].

Joint protection programs (JPP) are self-management strategies to help patients with hand OA preserve function and joint alignment. The primary principles involved in Joint Protection are the reduction of joint forces, reduction in joint deformity, and providing planning and pacing of activities. Originally shown to be effective for rheumatoid arthritis, this concept has been expanded to treat patients with Osteoarthritis. Joint protection programs involve training 'saver' movement patterns, the use of adaptive devices (i.e. built up handles, hands-free technologies) and behavior modifications (i.e. avoid type gripping). Current JPPs however are outdated ('use a pencil to dial a rotary phone'), have insufficient evidence to define best practice, are not definitively described and have widespread compliance issues [20].

\subsection{Biomechanical Models of the Hand}

Mechanical loading is believed to play a role in the development and progression of osteoarthritis[21]. Biomechanical models of the hand can be used in finite element analysis modeling to perform stress/strain analysis or in multibody segment simulation which enables inverse dynamic modeling and force dependent kinematic measurement to provide insight into normal and pathological biomechanics. Current models of the hand, however, are difficult due to the complex anatomy of the hand, and are lacking in their ability to accurately model mechanical interactions [22]. Many of these models rely on theoretical values of applied loads [23]-[27], and some rely on simple dynamometer measures of grip and pinch strength which do not accurately represent real world interactions [3]. These models of the hand would be greatly improved by the direct measurement of forces applied to the hand during function. 


\subsection{Current Methods of Hand Force Measurement}

Many measurement systems used to measure hand forces are do not directly measure these forces during activity. Current methods for determining forces in the hands typically involve either a dynamometer or some variation of a force glove $[1,4-6]$.

\subsubsection{Dynamometry}

The most commonly reported measures of hand forces are dynamometer-based measurements of grip strength or pinch strength [14]. These measures provide little insight into the forces required to complete various activities and are used clinically to determine maximum grip or pinch strength [28], [30]. While dynamometers provide a highly repeatable and accurate measure of hand force [4-5], they are unable to measure forces during a functional task [5].

\subsubsection{Sensorized Objects}

To solve the issues presented by dynamometry and force glove-based measuring systems, some researchers have embedded force transducers into devices that represent some common tasks [23], [24], [31]. Much work has been done to measure finger forces during specific activities by using instrumented objects (strain gauge/load cell) to measure applied loads exerted on objects while performing the functional tasks [23], [32], [33]. While this method allows for non-invasive and unobtrusive measurement of individual finger loads and can crudely simulate a small number of ADLs, it does not accurately represent real world activity, it is costly, and is limited in the types and number of functional tasks which can be examined/performed and cannot be used to measure forces during the actual performance of daily activities.

\subsubsection{Force Gloves / Sensors Attached to the Hand}

Alternatively, individual finger forces can be measured using force transducers that are attached to the fingers. This, however, often creates the issue of altering the contact between the volar surface of the hand and the surface being grasped and is not representative of the natural grip. Some sensorized glove constructs can measure forces in different finger segments and can be used during tasks of daily living [28], [29], [34]. However, sensor 
gloves typically occlude the surface of the volar dermis and do not allow for natural tactile feedback during activities [28], [29], [34]. Many of these constructs consist of flexible sensors which are sewn onto a leather or latex glove and significantly decrease the ability of the wearer to manipulate their fingers during activity.

\subsubsection{Strain Gauge and Capacitive Sensors}

Two of the most common transducers used to measure force in both sensorized objects and force gloves are strain gauges and capacitive sensors, each of which are capable of being calibrated with a load cell to measure forces [35]-[37]. Strain gauge transducers consist of patterned metal foil with flexible backing which attach to an object to measure deformation of the object [36]. As strain gauges are deformed, the metal coil's electrical resistance changes and this change in resistance is used to measure the amount of strain experienced by the object [28], [35], [36]. The biggest challenges with strain gauge sensors are their susceptibility to noise in the recording channel as well as being sensitive to fluctuations in temperature [36], [37]. Capacitive sensors, on the other hand, consist of two electrodes separated by a compressible dielectric matrix [38], [39]. As the sensors is compressed, the gap between the electrodes decreases and the capacitance changes [39]. Capacitive sensors are much less susceptible to noise and are not affected as much by temperature, however they are prone to failure in when shear forces are applied.

\subsection{Thesis Rationale}

Osteoarthritis (OA) is the most common disease of the joint and most frequently affects the IP and CMC joints of the hand [40]. Hand Osteoarthritis (H-OA) affects between 30 to $50 \%$ of the population over 45 years old [15], [18], [19], [41]-[43]. Unfortunately, H-OA has significant consequences including pain, loss of grip strength and limitations in hand function and participation. Joint protection programs are suggested for individuals with $\mathrm{H}$ OA to reduce load and effort during activities of daily living [20]. A reduction in the load and effort experienced by fingers, would in theory, reduce the strain on the joint structures which have been weakened by the disease and reduce pain and irritation and fatigue. Joint protection programs however lack evidence to support their use (few high-quality studies evaluating their effectiveness and are variable in description) and have low patient 
compliance as patients try to retrain their automatic movement patterns in daily activities to protect their joints and preserve hand function [20].

Technology has the potential to enact key JP principles of practice in context, repetitive training and immediate feedback. The overall objective of this thesis was to employ a wearable technology to collect information on hand forces on individuals with and without arthritis during activities of daily living involving the hand. In order to objectively quantify the reduction in hand forces that various JP principles attempt to achieve, a comprehensive examination of normal hand forces experienced during ADLs must first be examined. Using the wearable technology, it is then possible to examine the effect of H-OA on hand forces in individuals while performing hand related ADL's.

\subsection{Objectives and Hypotheses}

\section{Objectives}

1. Develop and validate method of measuring hand forces during activities of daily living.

2. Determine the envelope of applied forces by individual fingers during the performance of daily activities in healthy individuals

3. Determine the envelope of applied forces by individual fingers during the performance of daily activities in individuals with hand arthritis.

\section{Hypotheses}

1. A commercially available finger force measurement system will be durable enough to be worn by individuals while performing a set of activities of daily living and can be used to determine the forces applied by the hands during activity.

2. Based on previous literature which reported ranges of applied forces during activities of daily living using sensorized objects, the envelope of fingers forces is expected to be around 1 to $35 \mathrm{~N}$ [44]. 
3. Since individuals with hand arthritis commonly have a decreased grip strength and range of motion, it is hypothesized that individuals with arthritis would have a decreased envelope of applied forces during activities of daily living.

\subsection{Thesis Overview}

Chapter 2 describes the use of commercially available capacitive sensors to measure finger forces during the performance of activities of daily living. Results from a clinical study which examined 25 healthy participants and 21 participants with hand arthritis who performed 19 activities of daily living while wearing these sensors are shown and the forces are compared between the two cohorts. The capacitive sensors were then validated using the gold standard for force measurement, a single degree of freedom loadcell to determine the efficacy of using these sensors in this configuration.

Chapter 3 provides the conclusions of this work as well as future directions and applications of this research in the area of hand biomechanics. 


\section{Chapter 2}

\section{Individual Finger Forces during Activities of Daily Living}

This chapter explores the use of a commercially available force transducer system to measure finger forces during activities of daily living. Since these sensors had not been validated for use in this application, this chapter begins by performing testing to determine the accuracy and validity of these sensors (Objective 1). This chapter includes a study which measured finger forces during ADLs in healthy participants (Objective 2) as well as those with hand arthritis (Objective 3). ${ }^{1}$

\subsection{Introduction}

Pressure Profile Systems has created tactile sensors "Finger Tactile Pressure Sensors" (FingerTPS) which consist of small capacitive sensors sewn onto a micro spandex material that slips over the finger allowing for much more natural motion of the hands than previous force glove constructs [11]. Previous studies measuring hand forces at the finger joint using these sensors have examined a handful of specific tasks (such as handwriting, performing cardiopulmonary resuscitation, operating laparoscopic instruments during surgery, sport, and operating a drill) [38], [45]-[47]. While these measures are helpful in understanding forces during specific applications, an analysis of forces during a variety of common activities of daily living is needed. The objective of this research is to validate these sensors for the use in measuring hand forces during activities of daily living (Objective 1) and then to use these wearable force sensors to determine the envelope of applied forces during some common activities of daily living in healthy individuals (Objective 2) and those with osteoarthritis (Objective 3).

\footnotetext{
${ }^{1}$ A version of this chapter has been submitted for publication: Riddle, M, Robinson, S., MacDermid, J., Szekeres, M., Ferreira, L., Lalone, E. "Evaluation of Individual Finger Forces During Activities of Daily Living In Healthy Participants and those with Hand Arthritis." Journal of Hand Therapy: Innovation Special Issue. Invited Submission: May 2019.
} 


\subsection{Methods}

\subsubsection{TPS Sensors}

Finger Tactile Pressure Sensors (FingerTPS, Pressure Profile Systems, Los Angeles, CA, USA) were used to measure forces during activities of daily living. These sensors are made up of capacitive sensors which consist of two electrodes separated by a compressible dielectric matrix. As pressure is applied to the sensor, the distance between the electrodes decreases, and capacitance increases. These sensors were placed on the thumb and first three fingers (Figure 2.1). Each sensor was attached to a signal conditioning wrist module via a single wire and a $3.5 \mathrm{~mm}$ connection. This wrist module was connected to a wireless Bluetooth transmitter (D710 electronics interface module, PPS, Los Angeles, CA, USA) clipped to the belt or pocket of the participant which allowed the participant to move about the room freely during testing (Figure 2.2). Capacitance data from each sensor was received on a computer running proprietary Chameleon analysis software via a Bluetooth transceiver.

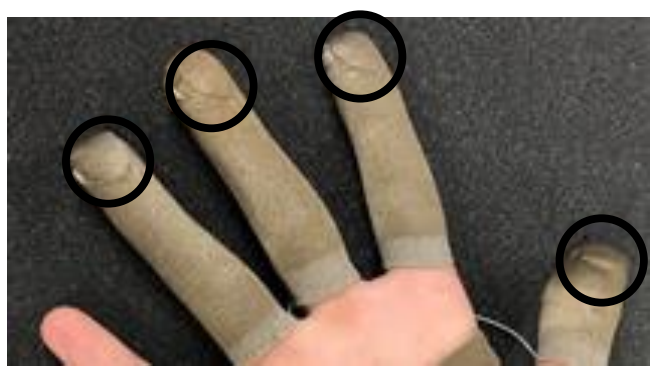

Figure 2.1 - Capacitive sensors are located at the fingertips (volar pad) of the thumb and first three fingers. 


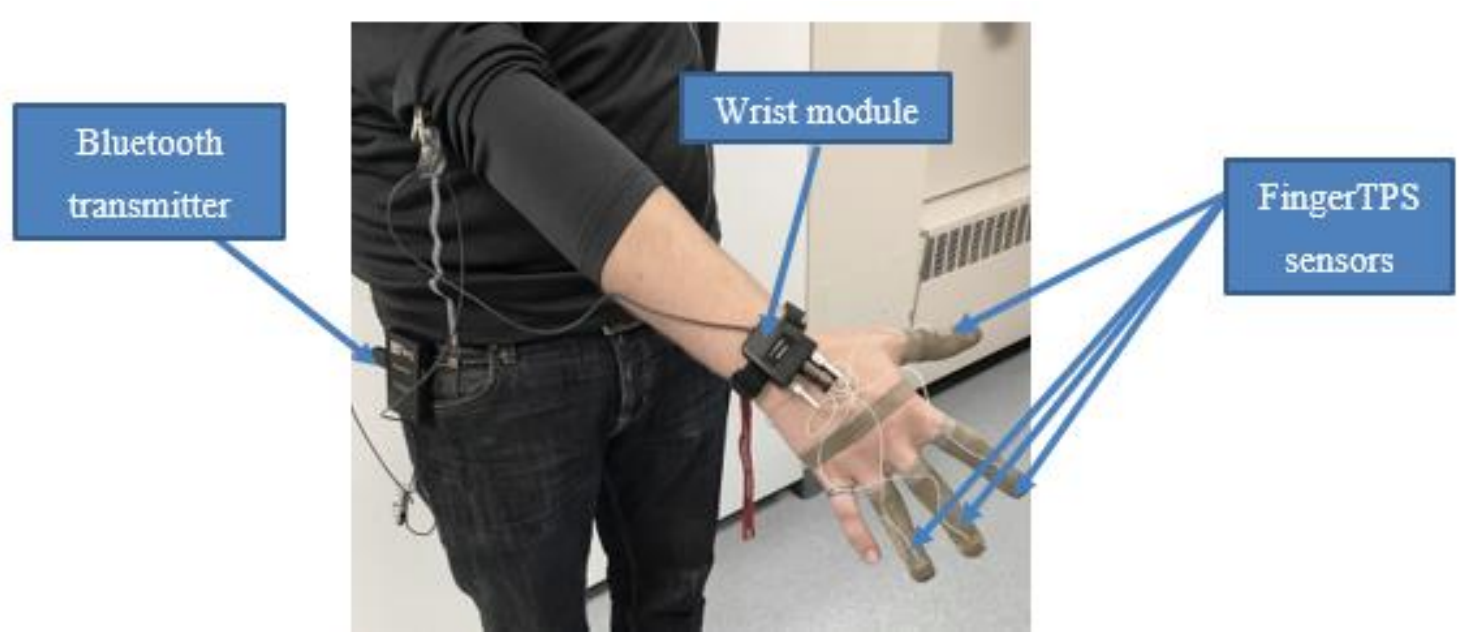

Figure 2.2 - Complete FingerTPS setup with four sensors (one each on the thumb and first three fingers), wrist module, and Bluetooth transmitter.

\subsubsection{Calibration and Use}

Each TPS sensor was calibrated to allow for the conversion from capacitance to force, measured in Newtons, N. In order to calibrate each sensor to measure force, the participant was instructed to press each finger on a load cell (Figure 2.3) (PPS, Los Angeles, CA, USA), gradually increasing the force until they reached a force of around 20N. Proprietary Chameleon Testing software (PPS, Los Angeles, CA, USA) then created a calibration equation to convert capacitance values from the sensors to force in Newtons. This process was completed by each participant for each of the sensor at the beginning of testing. 


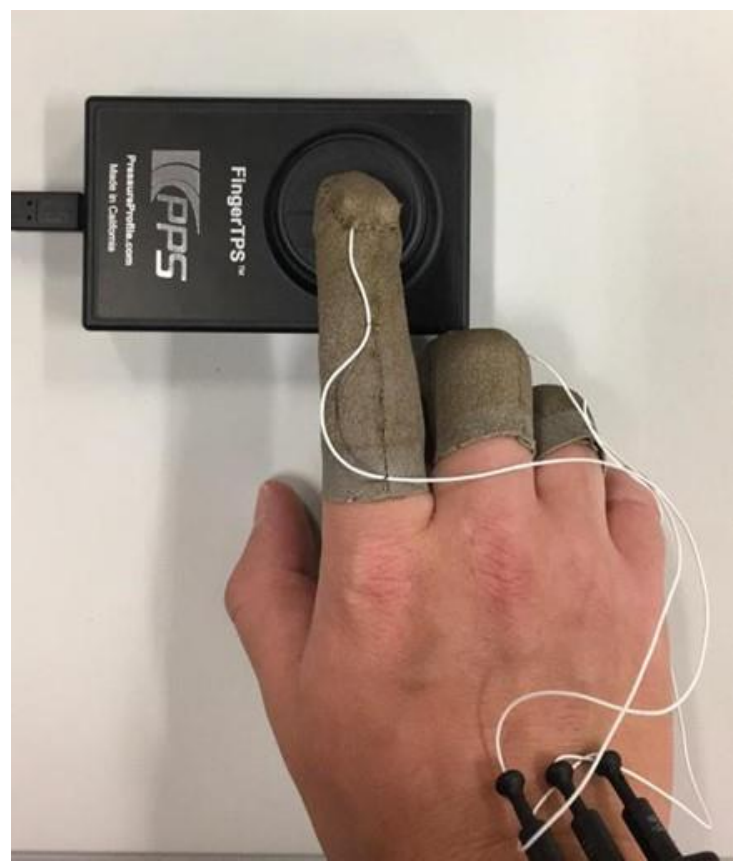

Figure 2.3 - Calibration of FingerTPS sensors using provided load cell.

\subsubsection{TPS Sensor Validation}

As these capacitive sensors have not been validated for use in the measurement of hand forces during activities of daily living, a series of analyses were performed to gain a better understanding of their strengths and limitations in this application.

\subsubsection{Load Cell Comparison}

To determine the accuracy of the calibrated FingerTPS sensors, the force output from the TPS sensors was compared to the force output of a clinical finger press load cell (model PF002, NK Upper Extremity Assessment System, NK Biomechanical Corp., Minneapolis, MN) as well as a Mini45 force/torque transducer (model SI-580-20, ATI Industrial Automation, Apex, NC).

\subsection{Accuracy and Repeatability of Calibration}

A single healthy participant (Male, 24 years) applied a total of 141 forces in a randomized order (one of five target forces: Light tap $[<0.5 \mathrm{~N}]$, Low $[\sim 5 \mathrm{~N}]$, Medium $[\sim 10 \mathrm{~N}]$, High $[\sim 15$ $\mathrm{N}$ ], Maximum [>15 N]) to a clinical finger press load cell (model PF002, NK Upper Extremity Assessment System, NK Biomechanical Corp., Minneapolis, MN) while wearing 
the FingerTPS sensors. The Finger TPS sensors were calibrated initially then removed and recalibrated after the $50^{\text {th }}$ and $100^{\text {th }}$ press of the loadcell. This allowed for the analysis of accuracy of the calibration when compared to a gold standard measurement as well as the repeatability of the calibration procedure.

The correlation coefficient between the two measurement systems was calculated for each of the three trials by creating a scatter plot with the loadcell output on the x-axis and the output from the FingerTPS sensors on the y-axis and a linear line was fitted to the data. A Bland-Altman plot was created for each trial to determine the agreement between the measurement systems. The average of the two measures was plotted on the $\mathrm{x}$-axis and the difference between them by subtracting the FingerTPS output from the loadcell output on the y-axis. The mean difference between the two measures, or the bias, was then plotted as a solid line. Finally, the limits of agreement were calculated as the mean $+/$ - two standard deviations.

\subsection{Drift over Time of Calibration}

Further testing was conducted to determine if the calibration holds true over an extended period while wearing the sensors continuously. To measure this, two FingerTPS sensors (one on the index and middle fingers) were calibrated as usual and the participant applied a series of 15 forces in a randomized order (three presses each of five target loads: Light tap [ $<0.5 \mathrm{~N}]$, Low [ $\sim 5 \mathrm{~N}]$, Medium [ 10 N], High [ 15 N], Maximum [ $>15 \mathrm{~N}]$ ), with each finger, to the load cell at three time points without recalibration. This was performed immediately after calibration, then again at 30 minutes and one hour. Between tests, the participant was free to use their hands. The difference between the FingerTPS sensors and the loadcell was calculated for each of the fifteen applied loads at each time point. The average difference between two was calculated at each time point and plotted along with a box-and-whisker plot to determine if there was an increase in variation with time.

\subsection{Effect of Shear Forces on Accuracy}

To determine the performance of FingerTPS sensors during shear forces, we measured shear forces applied by the finger in the $\mathrm{X}$-axis (left and right) and $\mathrm{Y}$-axis (forward and back) as well as the normal direction along the Z-axis. The same healthy participant 
performed a series of finger presses in each direction oscillating between the positive and negative direction while wearing the FingerTPS sensors. This was repeated twice for each of the three directions with the thumb, index, and middle fingers. Figure 2.4 shows the force measurement system used to measure these forces. A Mini45 force/torque transducer (model SI-580-20, ATI Industrial Automation, Apex, NC) was used to measure the applied forces in the $\mathrm{X}, \mathrm{Y}$, and $\mathrm{Z}$ directions.

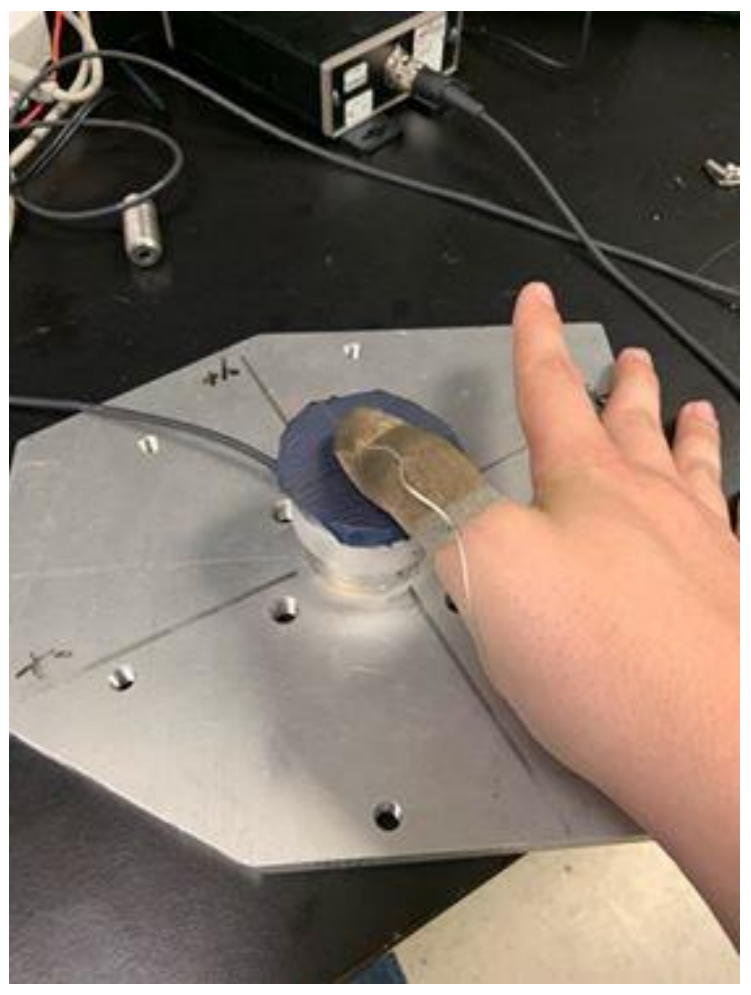

Figure 2.4 Shear force measurement setup.

\subsubsection{Dexterity Test}

In addition to testing the accuracy of the sensors when measuring force, it was of interest to determine the extent to which wearing these sensors altered the wearer's dexterity. Since these sensors slip over the finger and occlude the volar dermis, the natural tactile feedback of the hand is altered and the extent to which that alters the wearer's dexterity was unknown. In order to assess the change in dexterity, sixty participants were recruited to complete the N.K. Dexterity board [11] with bare hands and while wearing the sensors. The dexterity board, shown in Figure 2.5, consists of small, medium, and large objects such as 
blocks and spheres. In each subtest the participant manipulates the large, medium, or small objects [11], [12], [48]. Since hand dexterity involves the combination of different hand movements to efficiently manipulate objects, time is used as a measure of dexterity [11]. The order in which these subtests were completed, as well as if they were completed first with bare hands or while wearing the sensors, were randomized for each participant. Each subtest was completed three times in succession and the average time was recorded. The average time for all sixty participants was calculated for each subtest and the time to complete with bare hands and while wearing the sensors were compared to determine the effect of the sensors on hand dexterity.

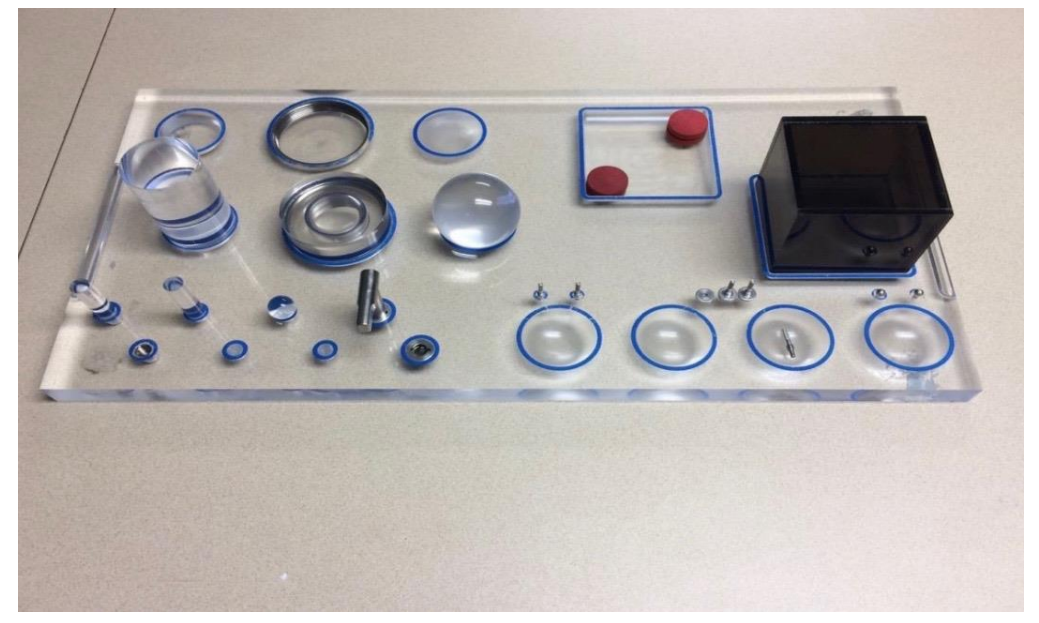

Figure 2.5. The N.K. Dexterity Board was used to assess the dexterity of individuals with bare hands and while wearing the FingerTPS sensors.

\subsubsection{Data Acquisition and Analysis}

For the load cell comparison tests for accuracy, repeatability, and drift over time, timestamped force $(\mathrm{N})$ data were recorded simultaneously from the single degree of freedom load cell and the FingerTPS sensors. For the shear force analysis, a six degree of freedom (6-DoF) loadcell was used to record time-stamped force $(\mathrm{N})$ data. The FingerTPS sensor data were collected at $40 \mathrm{~Hz}$ using proprietary Chameleon Testing software (Pressure Profile Systems, Los Angeles, CA, USA), and the load cell data were collected at $1000 \mathrm{~Hz}$ using LabVIEW 2018 (National Instruments, Austin, TX, USA). The FingerTPS system filtered the signal internally and the ADC resolution is reported to be 16 bits. No ad-hoc filtering was performed for either measurement system. The time-stamped force data were exported from each program to .csv files and custom written MATLAB (MathWorks, 
Natick, MA, USA) code was used to plot the force over time data for each trial and calculate the difference between both measurement systems as described for each analysis.

\subsubsection{Hand Force Measurement During ADL}

\subsubsection{Study Protocol}

Twenty-five healthy control subjects (12 female: 22-65 years old, 13 male: $20-53$ years old) were recruited for the study. Subjects were considered healthy if they reported having no pain, injury, or disease of the hand, such as arthritis. Additionally, 21 subjects with hand osteoarthritis (12 female: 52-79 years old, 9 male: 64-79 years old) were recruited for this study. These participants self-reported having been diagnosed with hand osteoarthritis. Participants were recruited from fliers posted on campus, at the Hand and Upper Limb Clinic, and in the local newspaper. Seventeen representative activities of daily living involving the hand were examined in this study (Table 2.1). These tasks were selected from some of the common tasks included in psychometric evaluations for individuals with hand/wrist pain including the Patient Rated Wrist and Hand Evaluation (PRWHE), Disability of the Arm Shoulder, and Hand questionnaire (DASH), and Joint Protection Behavior Assessment (JPBA) [49]-[51]. Tasks were selected from various aspects of daily life including kitchen tasks, cleaning tasks, dressing and grooming tasks. Further, these tasks were selected to include a combination of common power and precision grips to examine the effects of different grips on the forces exerted by the fingers. 
Table 2.1. The tasks from this study were selected from those commonly found on three psychometric evaluations: Patient Rated Wrist and Hand Evaluation (PRWHE), Disabilities of the Arm, Shoulder, and Hand (DASH), and Joint Protection Behavior Assessment (JPBA). Other ubiquitous tasks were added to our evaluation to get a better picture of the envelope of forces. * indicates task which were listed generically on the evaluation (turning on a tap and turning a doorknob) but were further specified for this study (lever vs. standard).

Task

PRWHE DASH JPBA

\begin{tabular}{|c|c|c|c|c|}
\hline 1 & Fill Mug & & & $\mathrm{X}$ \\
\hline 2 & Lift Mug to Mouth & & & $X$ \\
\hline 3 & Carry Empty Fry Pan & & & $x$ \\
\hline 4 & Cut Cucumber & $x$ & $\mathrm{X}$ & \\
\hline 5 & Open Water Bottle & & & \\
\hline 6 & Lift 2L Bottle & & & \\
\hline 7 & Open Jar & & $x$ & $x$ \\
\hline 8 & Standard Tap & & & $x$ \\
\hline 9 & Lever Tap & & & $x^{*}$ \\
\hline 10 & Open Pill Bottle & & & \\
\hline 11 & Push Plug Into Wall & & & $x$ \\
\hline 12 & Spray Bottle & & & \\
\hline 13 & Cut With Scissors & & & \\
\hline 14 & Write a Sentence & & $x$ & \\
\hline 15 & Standard Doorknob & $x$ & & \\
\hline 16 & Lever Doorknob & $\mathrm{X}^{*}$ & & \\
\hline 17 & Unlock Door with Key & & $x$ & \\
\hline 18 & Button Shirt & $x$ & & \\
\hline 19 & $\begin{array}{l}\text { Undo and Do Up a } \\
\text { Snap }\end{array}$ & & & \\
\hline
\end{tabular}

\subsubsection{Data Acquisition and Analysis}

Each subject performed each activity two times in the manner they would usually complete the activity. Including the set-up, and calibration, and performance of the activities, the total session duration was less than an hour and a half, with each task lasting only a few seconds. Time-stamped force $(\mathrm{N})$ data were sampled at a frequency of $40 \mathrm{~Hz}$. These data were recorded using proprietary Chameleon Testing software (Pressure Profile Systems, Los Angeles, CA, USA). These time-stamped force data were exported to a .csv file and custom 
written code (MATLAB, MathWorks, Natick, MA) was used to plot the force data over time and extract the peak forces for each finger during each trial.

\subsection{Results}

\subsubsection{FingerTPS Sensor Validation}

\subsubsection{Load Cell Comparison}

\subsection{Accuracy and Repeatability of Calibration}

A scatter plot with the $\mathrm{R}^{2}$ values and Bland-Altman plot comparing the loadcell and FingerTPS sensors output for each of the three trials are included in Figure 2.6. There was a strong correlation between the two measurement systems in all three trials with $\mathrm{R}^{2}>0.9$.

The agreement between the two systems was consistent for all three trials as well. The bias, or mean difference between the two measurement systems was $0.26 \mathrm{~N},-0.39 \mathrm{~N}$, and $0.51 \mathrm{~N}$ for each of the three trials, and the limits of agreement were $[-2.80,3.33 \mathrm{~N}],[-3.52,2.73 \mathrm{~N}]$, and $[-3.56,4.58 \mathrm{~N}]$. For each trial, $93 \%, 96 \%$, and $93 \%$ of the data points fall within the limits of agreement. In all three trials, the agreement between the two measurement systems appears to decrease with the magnitude of the measurement. 
A)
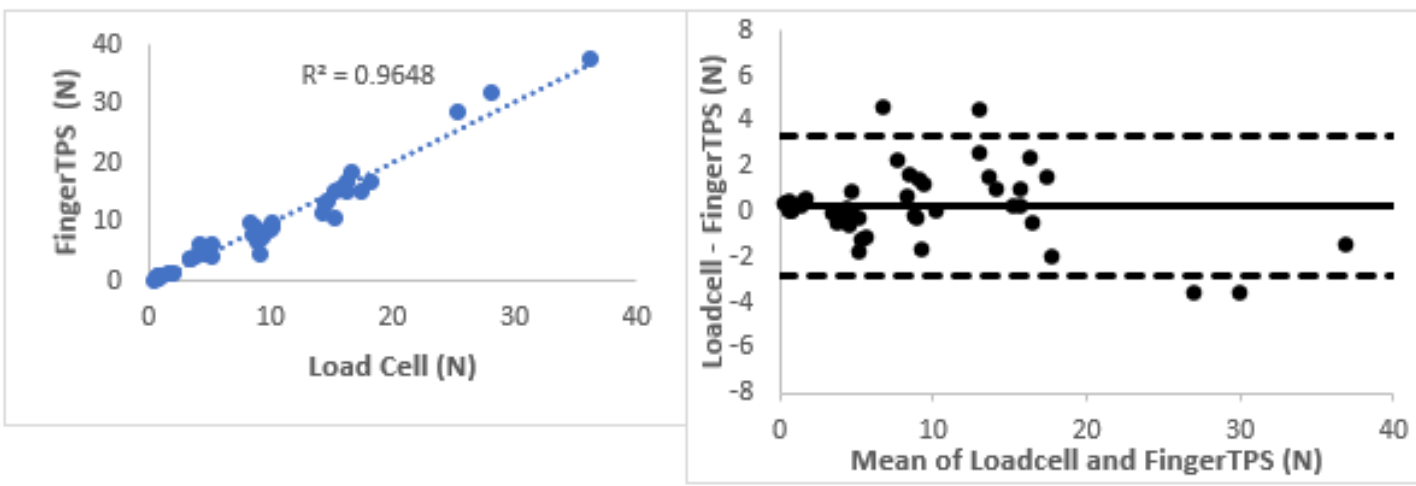

B)
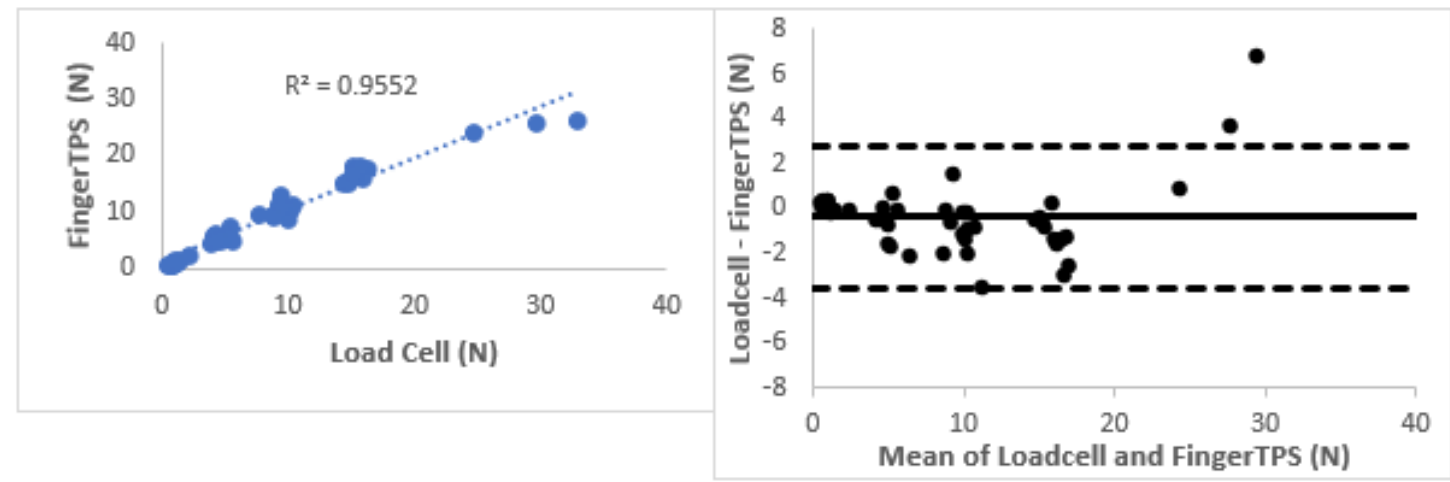

C)
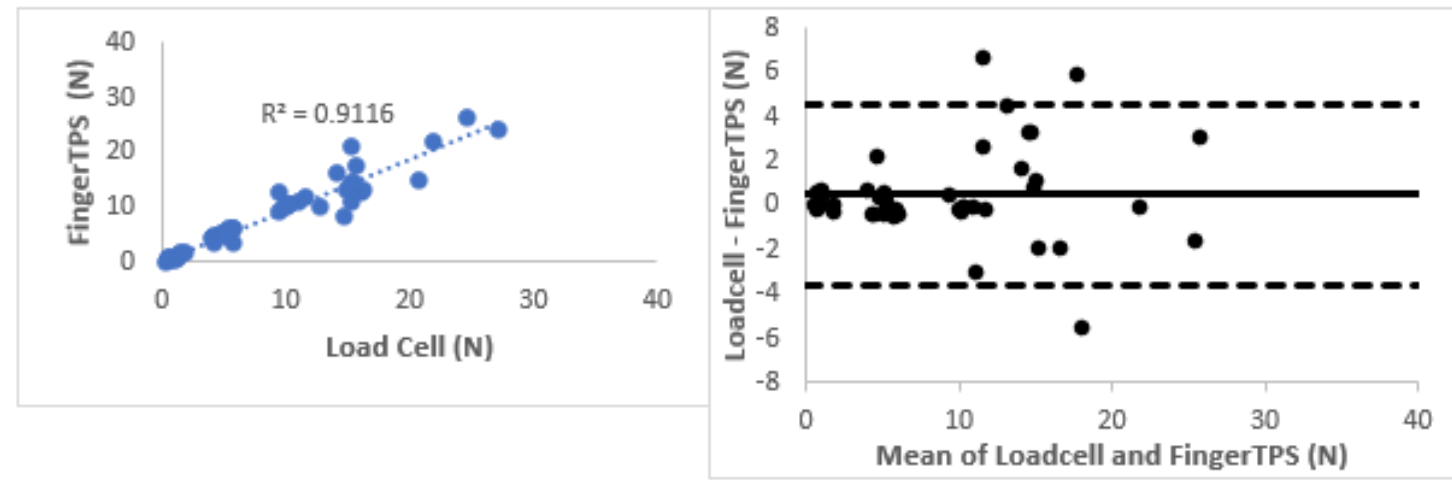

Figure 2.6 Scatterplot and Bland-Altman plots comparing the measurement of force from the FingerTPS sensors and Loadcell. A linear fit was used to calculate the correlation between the two measurement systems. For the Bland-Altman plots, the solid line represents the average difference, or bias, and the dashed lines indicate the limits of agreement. A) Trial 1, N=50; B) Trial 2, N=50; C) Trial 3, N=41.

To examine the distribution of the difference between the FingerTPS sensors and the load cell for all 141 forces, a frequency plot of these differences was created and can be found in 
Figure 2.7 below. The average absolute difference between the FingerTPS sensors and the load cell was $0.81 \pm 0.83 \mathrm{~N}[0-1.64 \mathrm{~N}]$.

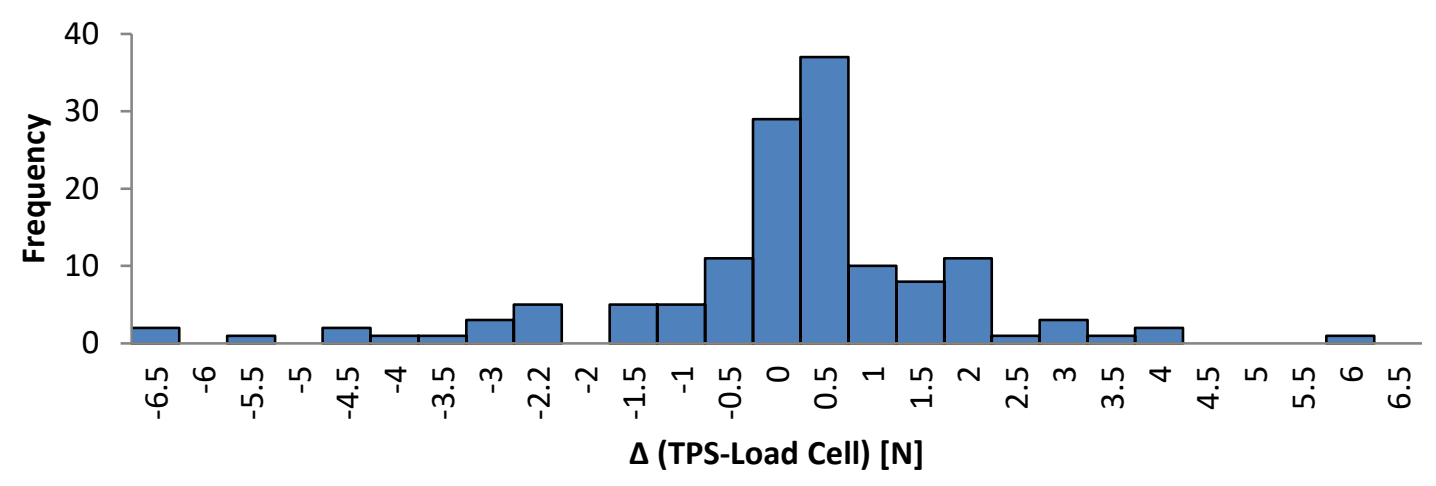

Figure 2.7. Frequency Plot showing the distribution of variation in recorded force value between FingerTPS and load cell. $(n=141)$.

\subsection{Drift over Time}

The force output from the loadcell and FingerTPS sensors for each of the 15 presses was recorded and the difference was calculated for each press. The average difference between the two measurement systems with a boxplot indicating the distribution of the differences is included below in Figure 2.8. 


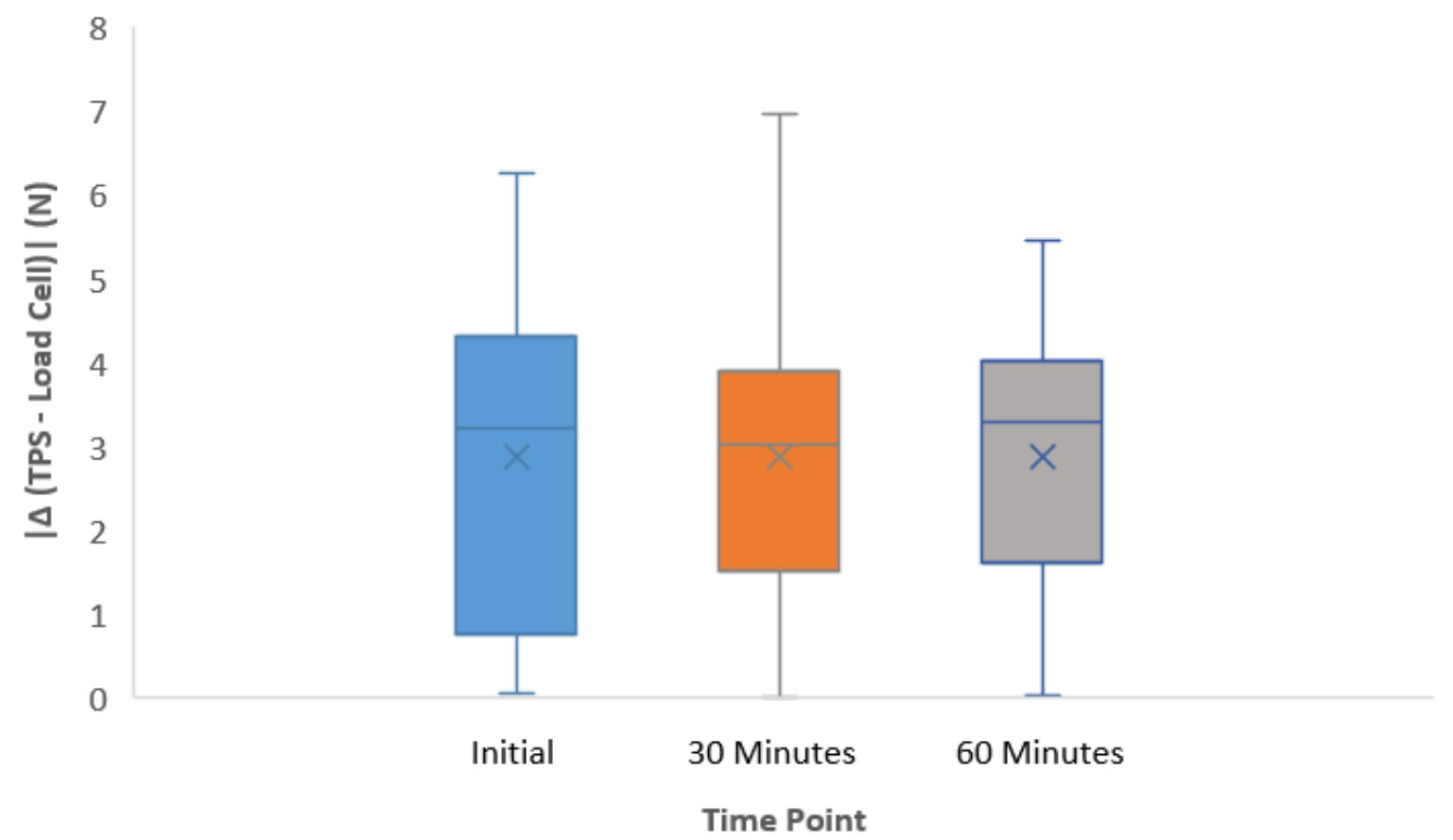

Figure 2.8. Boxplots indicating the spread of the differences at three time points to examine drift over time. The whiskers represent the maximum and minimum values. $X$ indicates the mean difference between the two measurement systems at each time point.

\subsection{Effect of Shear Forces}

Measurement from all three directions of the loadcell and the measurement of the FingerTPS sensors for shear in the $\mathrm{x}$-direction and $\mathrm{y}$-direction for two trials are plotted below in Figures 2.9 and 2.10. When shear forces are applied, the output of the FingerTPS sensors reflects elements of both the normal and shear forces and is larger than that measured by the loadcell. This suggest that these sensors are not capable of accurately measuring the applied forces when shear forces are present and will provide results which are larger than the applied normal force. Additionally, as seen in Figure 2.9, the signal from the FingerTPS sensors does not always return to zero when unloaded, which indicates that the shear forces may temporarily alter the calibration of the sensors. 

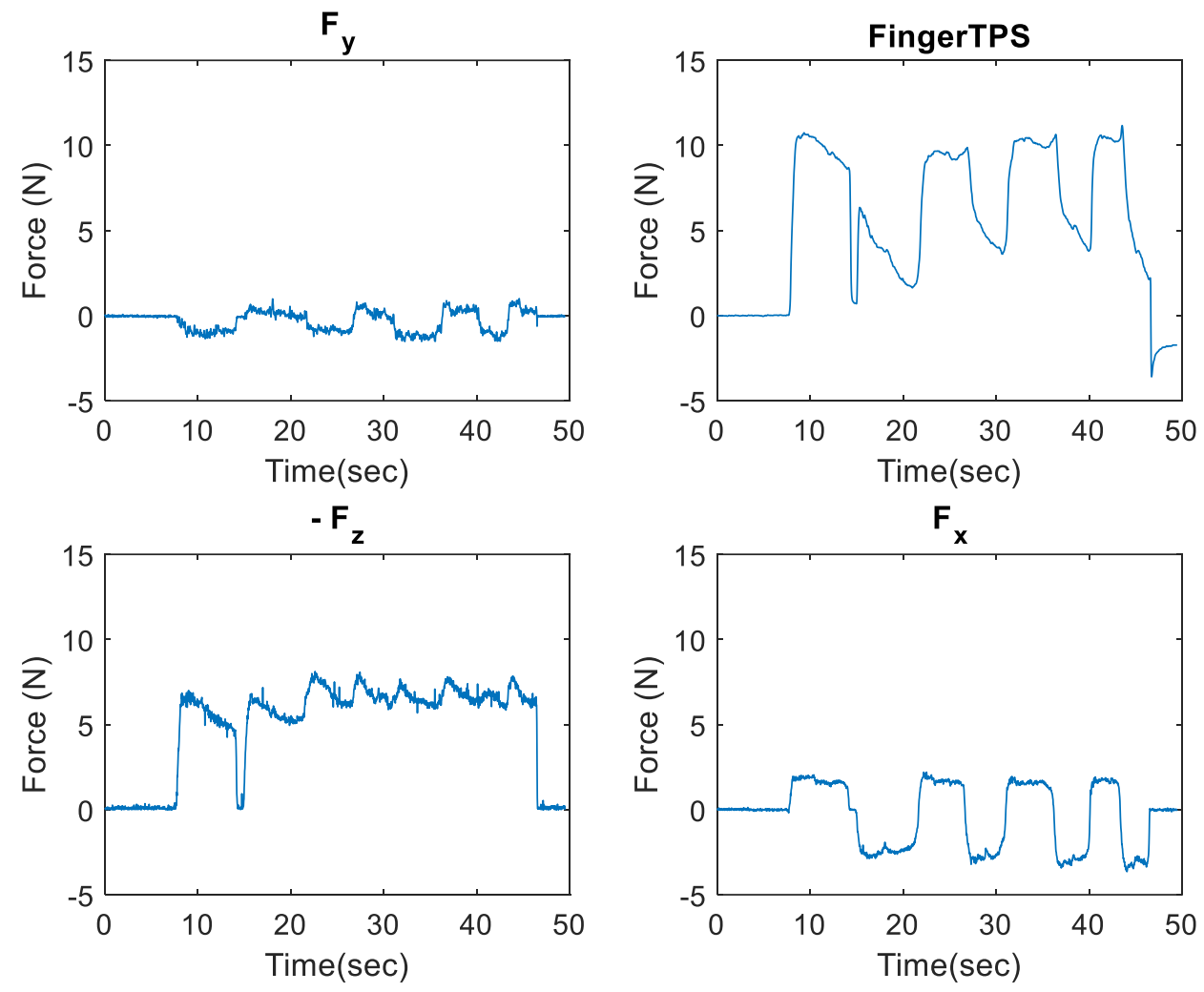

Figure 2.9 Example force over time for the loadcell in the $X, Y$, and $Z$ directions and the FingerTPS sensors when lateral (x-direction) shear is applied by the index finger. 

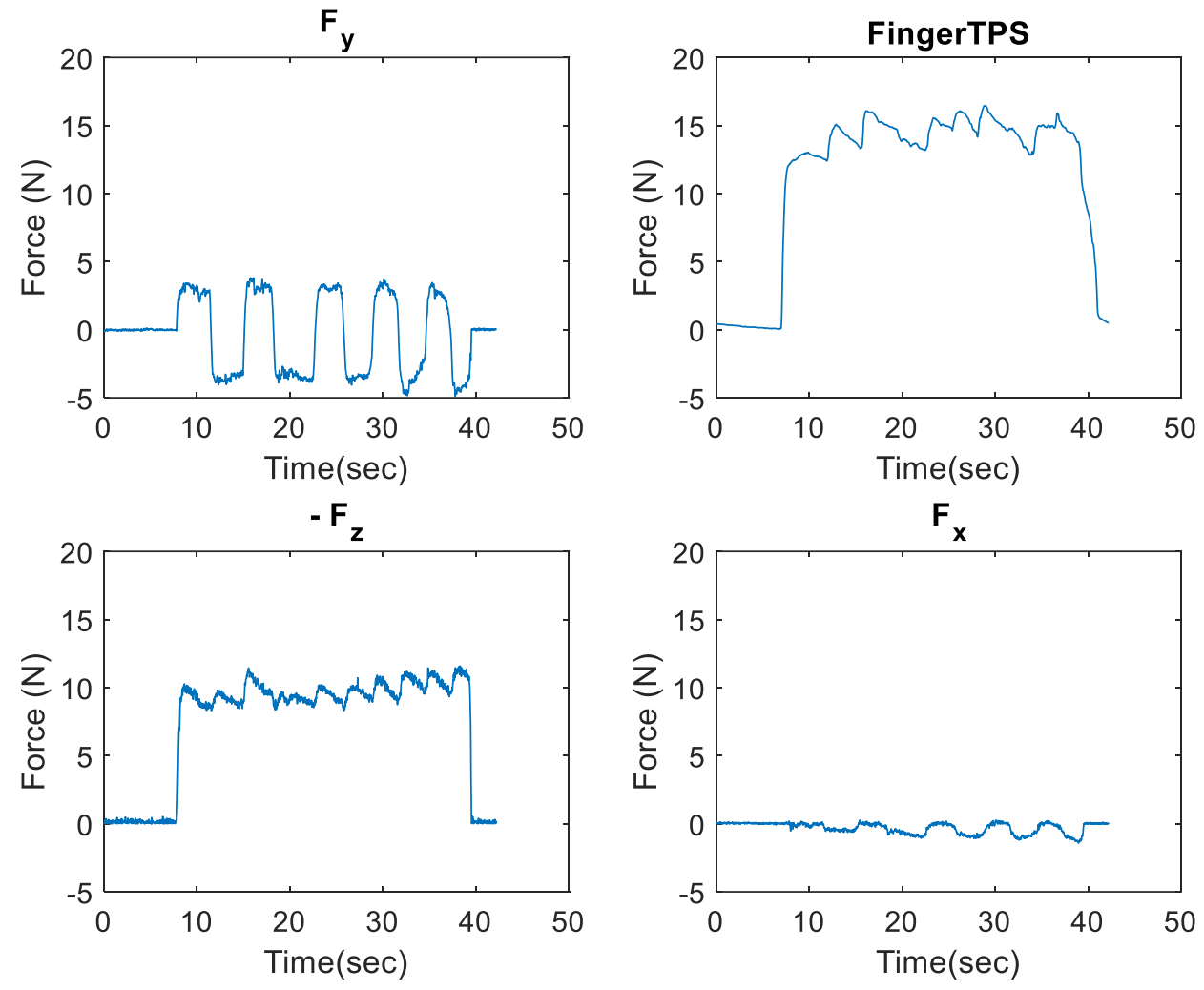

Figure 2.10 Force over time for the loadcell in the $\mathrm{X}, \mathrm{Y}$, and $\mathrm{Z}$ directions and the FingerTPS sensors when longitudinal (y-direction) shear is applied by the index finger.

\subsubsection{Dexterity}

In each subtest of the dexterity board, the time that it took to complete the test was significantly greater while wearing the sensors than with bare hands. This difference in time was the greatest in the small subtest, indicating that these sensors impede dexterity more for fine motor tasks. For the large subtest of the dexterity board, the time increased by 1.8 seconds, for the medium subtest 8.2 seconds, and for small 27.6 seconds. This increase in time was statistically significant for each of the three subtests. Figure 2.11 shows the average times along with standard deviation for each of the subtests of the dexterity board.

For the large subtest of the dexterity board, the time increased 1.8 seconds (13\%); for medium 8.2 seconds (31\%), and for small 27.6 seconds (41\%). Figure 3.7 shows the average times along with standard deviation for the dexterity board. 


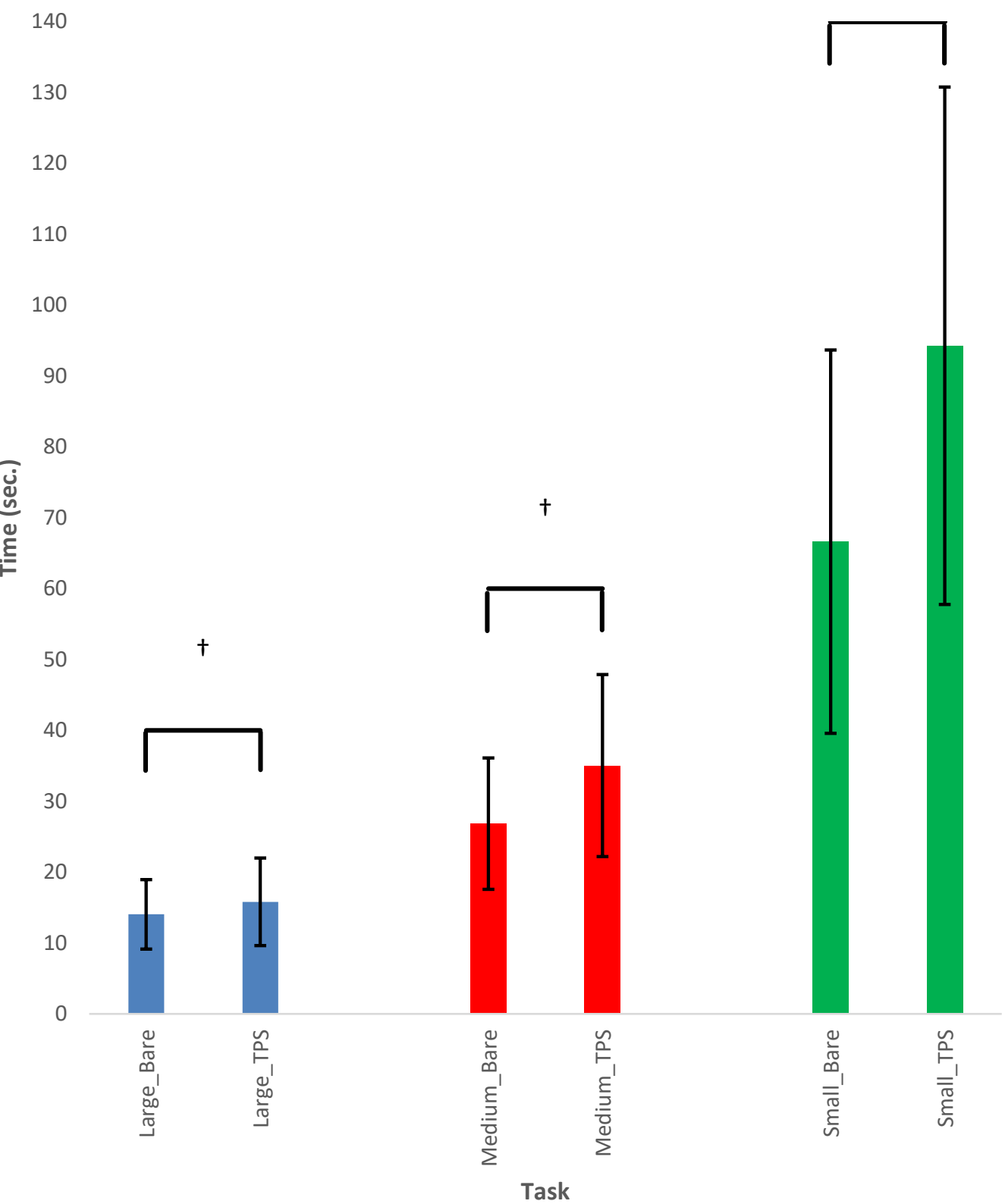

Figure 2.11. Average time and standard deviation to complete the dexterity board with bare hands and while wearing the TPS sensors. $n=60$ participants. $\dagger$ indicates a significant difference from a paired t-test. 


\subsubsection{Forces during Daily Tasks}

Maximum force during the performance of each task for each sensor was reported to make comparisons in the force data collected. The average maximum force was then calculated for each task and plotted for each sensor location (Figures 2.12-2.13). Standard error bars were used to indicate how far the sample mean is expected to be from the true population mean. Tasks were divided into three groups based on the primary fingers used to generate the force.

Tasks which primarily used the thumb and index finger in a precision grip included plugging in a toaster, opening a water bottle, opening a pill bottle, a snap button, turning a key in a door, buttoning a shirt and writing a sentence. These tasks are plotted together in Figure 2.12. The maximum force for these tasks ranged from $9.6 \pm 1.0 \mathrm{~N}$ to $34.8 \pm 1.6 \mathrm{~N}$ by the thumb during the shirt button task and the plug in task, respectively. Further, the smallest peak force was $1.4 \pm 0.6 \mathrm{~N}$ by the ring finger during the key turning task. For participants with osteoarthritis, the maximum force ranged from $7.9 \pm 1.8 \mathrm{~N}$ by the thumb during the shirt button task and $30.7 \pm 3.7 \mathrm{~N}$ by the thumb during the plug in task, while the smallest measured peak force was $2.9 \pm 0.7 \mathrm{~N}$ by the ring finger during the writing sentence task. 

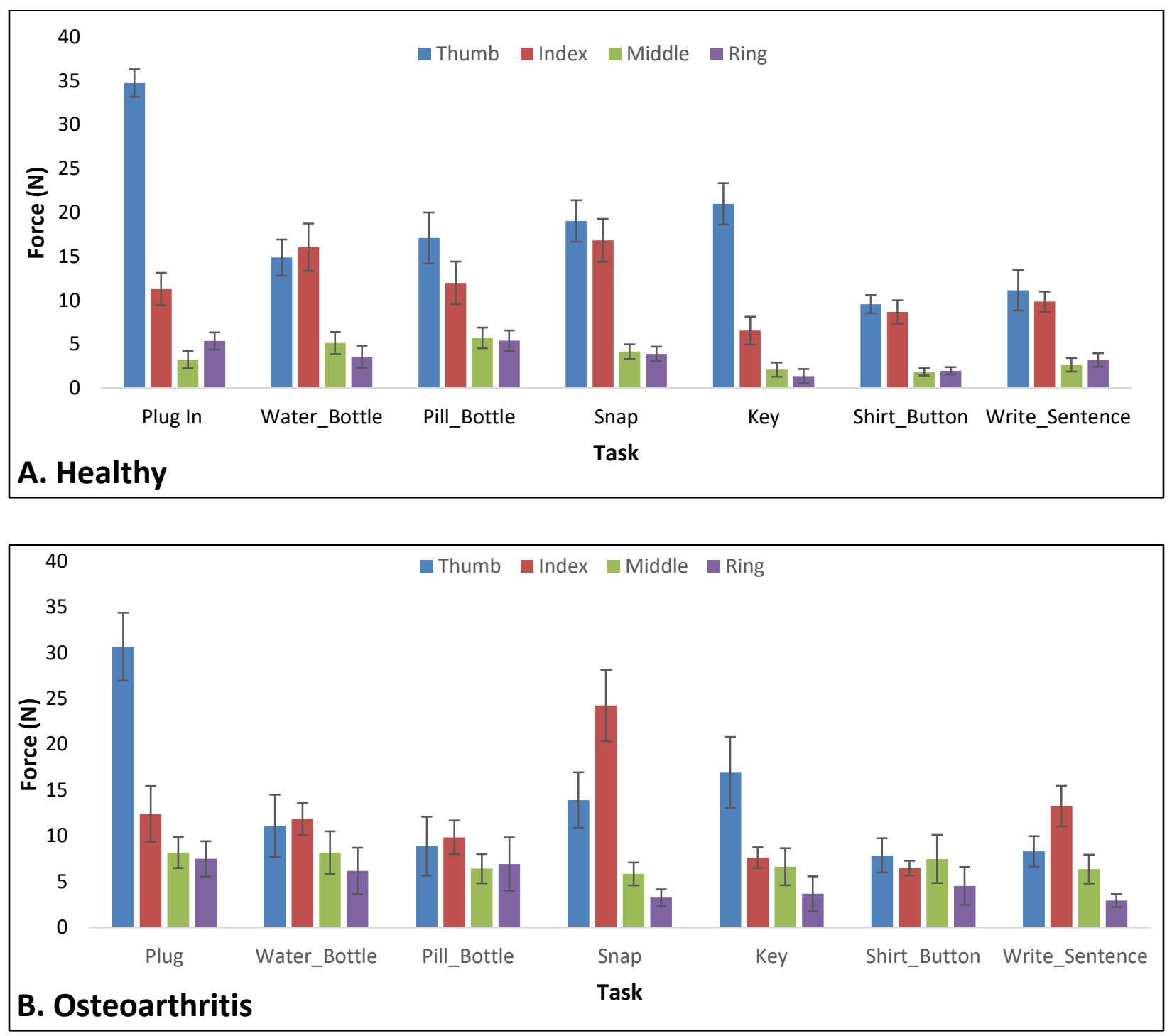

Figure 2.12. Max force graphed for each finger in Newtons with standard error for tasks dominated by the thumb and index finger (precision grip). A. $n=25$ healthy participants. $B . \mathbf{n = 2 1}$ participants with osteoarthritis.

Tasks which utilized a power grip, using all four fingers to apply the force, included pouring a kettle, lifting a mug to mouth, carrying a frying pan, cutting a cucumber, lifting a $2 \mathrm{~L}$ bottle, cutting with scissors, and opening a jar. The average maximum force applied by each finger during these tasks are plotted in Figure 2.13. The average maximum force for these tasks in healthy participants ranged from $4.4 \pm 1.8 \mathrm{~N}$ to $19.7 \pm 2.7 \mathrm{~N}$ by the ring finger during the scissors task and thumb during the cutting cucumber task, respectively. The lowest peak force for these tasks was $3.2 \pm 0.7 \mathrm{~N}$ by the index finger during the scissors task. For participants with osteoarthritis, this range was $4.8 \pm 1.4 \mathrm{~N}$ to $15.4 \pm 3.2 \mathrm{~N}$ by the 
index finger during the scissors task and the middle finger during the pouring kettle task, respectively. This lowest peak force for participants with osteoarthritis was $2.3 \pm 1.0 \mathrm{~N}$ by the thumb during the scissors task.

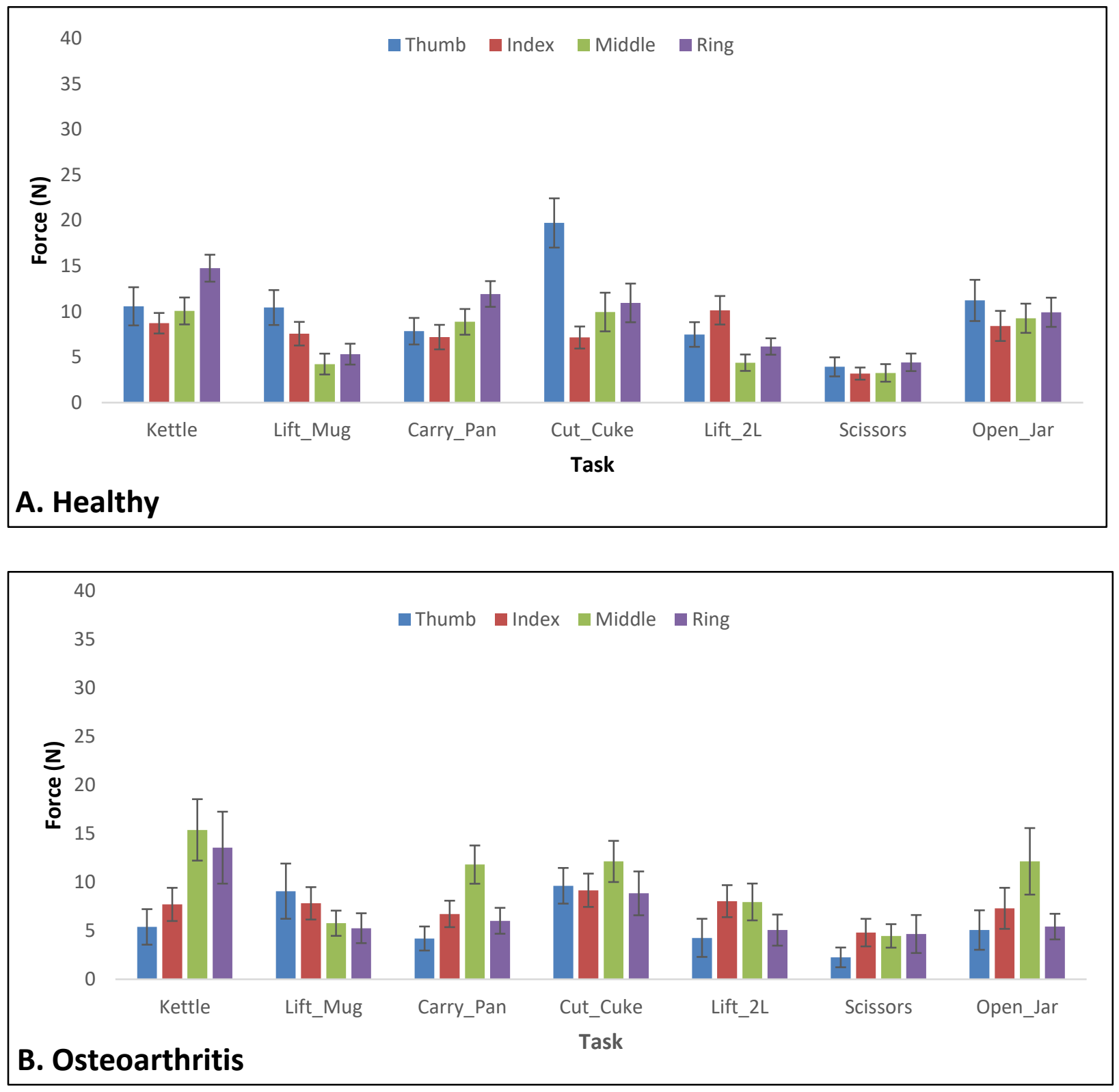

Figure 2.13. Max force graphed for each finger in Newtons with standard error for tasks that rely on all four fingers (thumb, index, middle, ring) to generate force (power grip). A. $n=25$ healthy participants. $B$. $n=21$ participants with osteoarthritis.

Tasks which didn't clearly fall into either the precision grip between the thumb and index finger or the power grip with all four fingers included the spray bottle and both the standard 
and lever tap and doorknob. The average maximum forces for these tasks are plotted in Figure 2.14. The task with the largest magnitude of force for the healthy participants was the standard tap at $13.0 \pm 2.5 \mathrm{~N}$ by the thumb. However, for participants with osteoarthritis, the task with the largest peak force was the spray bottle which was $16.1 \pm 2.7 \mathrm{~N}$ by the middle finger.
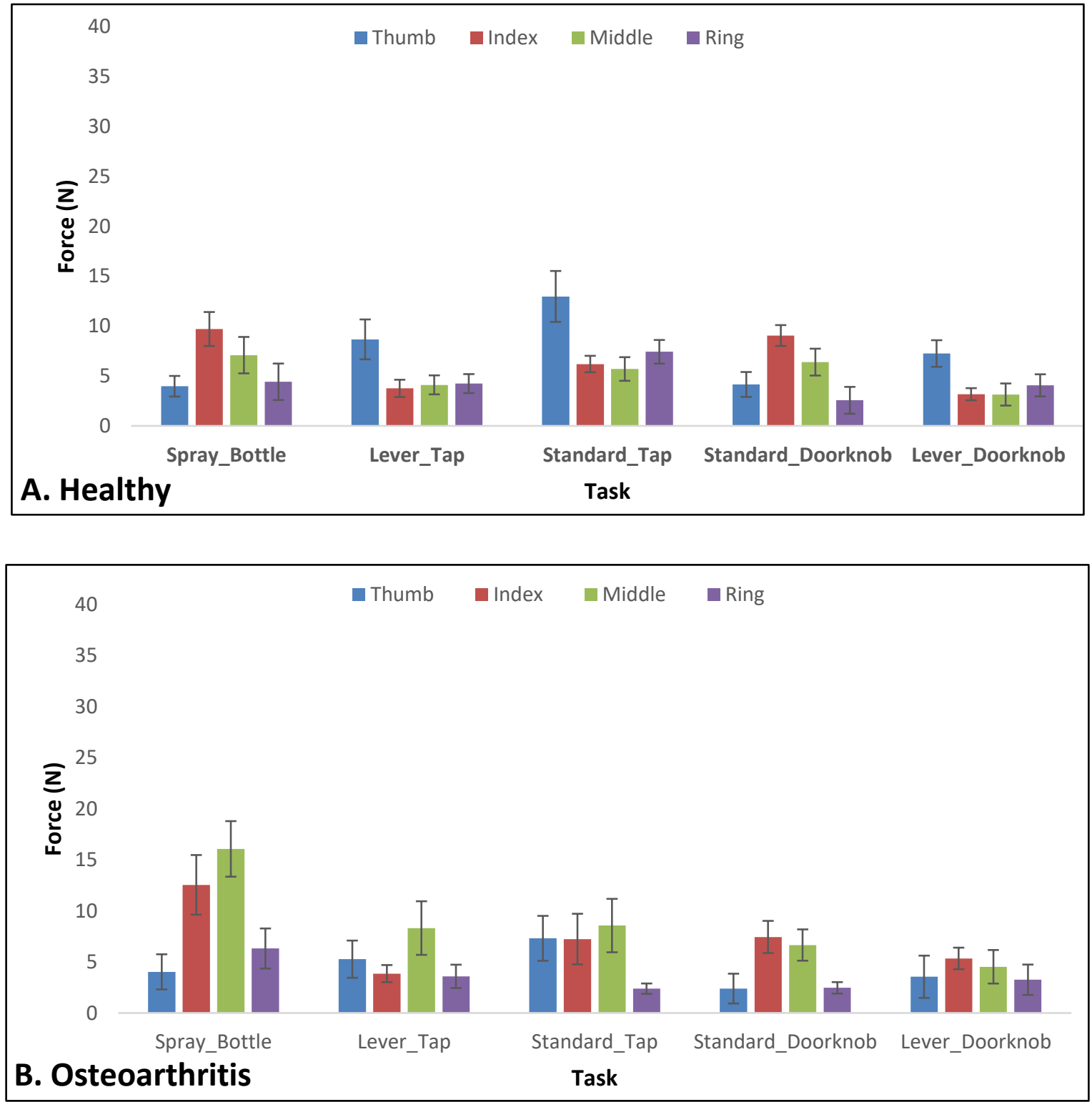

Figure 2.14. Max force graphed for each finger in Newtons with standard error for tasks with some other combination of primary movers (power and precision grips). A. $n=25$ healthy participants. $B$. $n=21$ participants with osteoarthritis. 


\subsection{Discussion}

The FingerTPS sensors allowed for the measurement of individual finger forces during the activities examined. They were capable of isolating grips based on finger recruitment for various tasks and providing insight to the magnitude of forces in each finger during activity. The envelope of applied forces during these activities of daily living in healthy participants was between $1.4 \pm 0.6 \mathrm{~N}$ to $34.8 \pm 1.6 \mathrm{~N}$ (in the ring finger when turning a key and the thumb when plugging a cord into an outlet, respectively). This envelope of forces varied slightly in participants with osteoarthritis. For this group of participants, the envelope of applied forces was $2.3 \pm 1.0 \mathrm{~N}$ to $30.7 \pm 3.7 \mathrm{~N}$ (in the thumb during the scissors task and the thumb during the plug in task, respectively). This envelope, for both the healthy participants and those with osteoarthritis, is consistent with that measured by the Department of Veterans Affairs (VA) in a similar study which measured the forces required to complete 12 activities of daily living. This study reported the envelope to be between 1.4 $\mathrm{N}$ and $31.4 \mathrm{~N}$ (in a push button remote task and plug in task, respectively) [44].

Compared to published normative pinch strength data for healthy adults, the envelope of applied forces during these activities falls well below the average maximum pinch strength. This indicates that participants are generally able to generate a much higher force than was required to complete activities of daily living. Werle et. al. published a study in 2009 which reported normative pinch and grip strength of 1023 healthy adults in 5-year age brackets [52]. In 299 healthy men between the ages of 20 and 64, the range of maximum pinch strength was $60.8 \mathrm{~N}-134.4 \mathrm{~N}$, with an average of $98.3 \mathrm{~N}$. For the same measure in 304 women in this age bracket, this range was $31.4 \mathrm{~N}-122.6$, with an average of $67.8 \mathrm{~N}$. For men and women combined, the range of maximum pinch strength would be $31.4 \mathrm{~N}-134.4$ $\mathrm{N}$ with an average of $82.9 \mathrm{~N}$.

There was a strong correlation between the FingerTPS sensors and the loadcell when normal forces are applied by the hand. This was consistent over three trials indicating the calibration technique is repeatable. When examining the agreement between the two measurements, an inverse relationship between the magnitude of the measurement and the agreement was noted. This suggests that the accuracy of the FingerTPS sensors depends on the magnitude of applied force. There were some outliers in the Bland-Altman plots noted in 
each of these trials. More than three unexplained outliers per 100 measurements is the threshold which indicates a problem with a measurement system [53], [54]. In each of the three trials there were roughly twice this number of outliers, however they are likely the result of off-axis loading (i.e. shear). When all three trials are combined, the average difference in force measurement between the TPS sensors and the load cell was $0.81 \pm 0.83$ $\mathrm{N}$. This difference is within one standard error for most of the activities examined, suggesting that measuring these forces using the FingerTPS sensors is a valid technique. However, as noted, when off-axis loading occurs, the relationship between the FingerTPS sensors and a loadcell is not consistent. This suggest that these sensors are not suitable for measuring activities where shear forces are present. Dexterity was impeded in each subtest of the dexterity board, but was less impeded during the large and medium grip tasks when compared to the small grip tasks. However, all of the activities examined in this study would be classed as large or medium grip tasks.

\subsubsection{Limitations}

There were some limitations found while using the FingerTPS sensors. Since these sensors are propriety and can only be purchased from the manufacturer, they can only be used with their propriety Chameleon Testing Software (Pressure Profile Systems, CA). This greatly decreases the setup time required to use the system and makes the sensors inherently more user friendly, however doesn't allow for external validation of the calibration equation generated when calibrating these sensors. However, from repeated testing with a load cell and calibrated sensors, it is possible to determine the accuracy of the calibration, so this is not a major limitation. Another limitation from the setup used for this study was that we were only able to measure forces at the fingertips, losing valuable information about the applied forces to various finger segments, namely the middle and proximal phalanges of each finger. The manufacturer does sell sensors for these finger segments and future work should include these sensors. Finally, a limitation of these capacitive sensors is their inability to accurately measure forces when shear forces are present. Though many of the activities performed are thought to be primarily normal forces, it is uncertain the extent to which shear forces may have altered the force measurements recorded. This is likely most 
relevant in tasks such as opening a jar and standard doorknob where torsion is required to complete the activity.

Further, there were limitations in the study design and sampling as well. While this study sought to select ubiquitous tasks that represent daily life, this study only examined 19 activities of daily living which may not give an accurate representation of the true envelope of forces. There was a large disparity in age between the two cohorts, with a generally young sample of healthy individuals and a much older sample of participants with arthritis, which is likely a compounding factor in the difference in force reported as grip strength tends to decrease with age. Additionally, we did not use a scale to grade and record the type, location, and severity of osteoarthritis present in the individuals with arthritis. As reported, there were 21 individuals with hand arthritis included in this analysis, however there were initially 35 recruited to participate. Fourteen of these individuals were unable to complete many of the ADLs due to pain and were therefore not included in the data analysis. As a result, the reported envelope of applied forces for individuals with hand arthritis does not include those with severe symptomatic OA. In the preliminary stages of this research, we made an effort to include a patient-consumer to determine if the selection of ADLs was appropriate for individuals with arthritis. However, this patient-consumer was high functioning as a result of their arthritis and in the future, there should be patient-consumers who represent the entire scale of functional ability.

\subsubsection{Future Work}

Further measurements of forces applied by the fingertips would add to the accuracy of current biomechanical models of the hand and measuring the forces in other finger segments would shed valuable insight as well. For tasks which use a hook grasp or similar grasps, the majority of the applied force is exerted by the middle phalanges and only measuring the finger tips in such activities misses some valuable data.

Unlike the lower extremity that has the standard gait cycle, there is not standard functional assessment that can be used to assess function. In this study, we propose a set of activities of daily living that are based on current patient reported outcomes and functional assessments and can be used to create a standard 'functional assessment' of the hand and upper limb. To 
limit the effects of coaching on the exertion of force, participants in this study received no instruction on how to perform each activity. However, it is uncertain the degree to which different grips/methods of performing these activities alters the magnitude of force. A thorough analysis of these variations in grip and methods of performance should be conducted. Future work should examine forces applied by the hand during other applications such as recreation, sport, and vocational (work) tasks. Similarly, the relationship between reduced grip strength and the ability to perform activities of daily living as well as the applied forces in the hand during these activities should be examined. This study has shown that by using FingerTPS sensors to measure tactile forces, we are able evaluate individual finger forces during activities of daily living and gain valuable insight into both the magnitude of forces and the recruitment of fingers during activity. 


\section{Chapter 3}

\section{General Discussion and Conclusion}

This chapter reviews the objectives and hypotheses presented in the thesis, discusses the implications, strengths and limitations of the research, and outlines future work that should follow in future studies.

\subsection{Summary}

Hand Osteoarthritis (H-OA) is the most common disease of the synovial joints of the hand and effects nearly half of the adult population over the age of 45 [15], [16], [18]. Currently, there is no known cure for osteoarthritis and treatments are limited [16], [17]. In order to assess the effects of H-OA on hand function and the effectiveness of JPP at reducing these forces, it is necessary to examine normal hand forces during activities of daily living (ADL). The overall objective of this thesis was to employ a wearable technology to assess hand forces in healthy individuals and those with arthritis during ADLs involving the hand. This thesis examines the use of two different wearable technologies to assess hand forces during ADLs and presents an envelope of applied forces for both healthy individuals and those with hand arthritis.

This thesis examined the use of one of the commercially available measurement systems to measure these forces (Finger Tactile Pressure Sensors, FingerTPS, Pressure Profile Systems, CA). While these sensors consisted of transducers placed on the volar dermis of the hand which alters the natural grip of the hand, the material used in these sensors allowed for much more motion than others on the market. Further, they were marketed for measuring force during sport applications, so they were expected to be robust enough to measure forced during activities of daily living. This study attempted to address all three objectives of this thesis. Since these sensors had not previously been used in this application, work was done to develop protocols to use these sensors to measure hand forces in a series of functional tasks. Subsequent to this, the validity of using these sensors to measure forces during ADL was examined by comparing the measurement to a gold standard (Objective 1). In this study, participants with and without hand arthritis were recruited and asked to 
complete 19 ADLs while wearing these sensors to determine the envelope of applied forces experienced while performing these ADLs (Objectives 2 and 3). Ultimately these sensors were found to be suitable for this application (Objective 1) and were successfully used to measure the envelope of applied forces in both healthy participants and those with arthritis (Objectives 2 and 3, respectively). Further, as hypothesized (Hypothesis 2), the values measured for healthy participants were consistent with prior work and there was a measurable decrease in these forces for individuals with arthritis, though there were confounding factors described in Chapter 2, so Hypothesis 3 was partially supported.

\subsection{Significance}

Measurement of the magnitude of forces exerted by the fingers during activities of daily living and how these forces change for individuals with pathologies such as arthritis is valuable to our understanding of how the hands function and how hand function is affected by disease. This study serves as a precursor to a larger study looking at JPP and consists of an initial description of this technology and the development of the possible protocol to be used. This work represents the first step in assessing the effectiveness of JPP at reducing forces for individuals with H-OA. Having examined the normal forces experience during ADLs, researchers can determine, objectively, if enacting key JP principles reduces hand forces. Additionally, the ability to directly measure these hand forces for individual fingers in a broad array of activities has the potential to greatly improve current biomechanical models of the hand which will, in turn, allow for a greater understanding of the internal joint loading experienced during activity. This hand force information is also vital to the development of upper limb prosthetics. A major limitation in the current design of hand prosthetics is a lack of information about the amount of force required to complete various tasks [55], [56].

\subsection{Limitations}

It is noted that there are limitations to the studies presented in this thesis. A major limitation to the study design in Chapter 2 is that there was a disparity in age between the healthy individuals and those with hand arthritis which was a major confounding factor that confounded direct comparisons between these two groups. Additionally, as a result of 
convenience recruitment used to recruit participants for the study, the sample size for both groups were relatively small. Further, many of the individuals with arthritis recruited to participate in the study were unable to complete all of the ADLs due to pain and therefore were not included in the data analysis. Another major limitation of this work is the fact that the severity of hand arthritis in the individual recruited was not factored into the analysis of hand forces.

Several limitations of the technology used were discussed in Chapter 2. Namely, the material used in these sensors and placement of the sensor on the volar surface of the hand impede dexterity and alter the natural tactile feedback of the hand, and capacitive sensors inability to accurately measure off-axis loads which occur during shear.

Despite these limitations, there were several strengths of this work as well. All three objectives of this thesis were addressed. The sensors described in Chapter 2 were found to be capable of measuring forces applied by the fingers in this application and were able to be used to successfully determine the envelope of applied forces during activity. The results were consistent with prior work, suggesting these measurements are reasonable. This work has also created repeatable protocols which can be used to expand the measurement of finger forces.

\subsection{Future Directions}

The results of this research have established a starting point for future studies. Firstly, in the short term, efforts should be directed toward increasing the sample size for both the healthy and arthritic participants, while age matching the healthy participants. This larger sample would allow for the examination of the effect of age, sex, and severity of arthritis on hand forces. After evaluating finger forces during the performance of ADL in individuals with and without hand arthritis (Chapter 2), the next step will be to evaluate how these forces are altered when JPP are employed. While this thesis focused on the envelope of applied loads by the finger by examining the peak forces applied by each finger, future work should look at other aspects of the force measurement as well, such as the total force applied while completing activities as well as the time it takes to complete various ADL. 
Another area that should be explored from the results of this study and continued collection of force data to follow is importing this force data into biomechanical models of the hand to examine internal loading of the joints during these activities. Combined with kinematic data collected in our lab during the performance of the same ADLs, a complete picture of joint loading could be acquired using current biomechanical hand models. These models would also help answer the question. It has been suggested that the aim of JPP in individuals with $\mathrm{H}-\mathrm{OA}$ is to reduce joint loading on articular cartilage, strengthen muscle support, and improve the shock-absorbing capabilities of joints [20], [57], [58]. Since the aim of JPP is to reduce loading on articular cartilage, it would follow that the hand forces should be decreased when these principles are employed and biomechanical models would allow for insight into changes in joint loading.

Another area of interest is using this wearable technology as a training tool for individuals with hand arthritis to help improve compliance of JP principles. One possible method of achieving this would be the implementation of a biofeedback system (a sound or a light) to alert the wearer when high forces are exerted. This would help to prevent patients from "pushing through" during the performance of an activity which can have detrimental effects to the joints of the hand.

Finally, other measurement technologies should be pursued for this application which may perform better when shear forces are applied and do not limit the wearer's dexterity as much as the FingerTPS sensors. One promising sensor was recently introduced by IBM which use strain gauge technology and accelerometers on the dorsal aspect of the fingers to measure forces and other biomechanical information during activity [59]. 


\section{References}

[1] G. D. Kessler, L. F. Hodges, and N. Walker, "Evaluation of the CyberGlove as a whole-hand input device," ACM Trans. Comput. Interact., vol. 2, no. 4, pp. 263-283, 1995.

[2] A. M. Dollar, "Classifying Human Hand Use and the Activities of Daily Living Activities of daily living,” 2014.

[3] J. L. Joaquín L. Sancho-Bru, Antonio Pérez-González, Marta C. Mora, Beatriz E. León, Margarita Vergara and P. J. R.-C. and A. M. Iserte, "Towards a Realistic and Self-Contained Biomechanical Model of the Hand," Theor. Biomech., pp. 211-240, 2011.

[4] M. H. Schieber and M. Santello, "Neural Control of Movement Hand function : peripheral and central constraints on performance," J. Appl. Physiol., vol. 96, pp. 2293-2300, 2004.

[5] W. Chen, C. Xiong, X. Huang, R. Sun, and Y. Xiong, "Kinematic analysis and dexterity evaluation of upper extremity in activities of daily living," Gait Posture, vol. 32, no. 4, pp. 475-481, 2010.

[6] J. Hamill and K. M. Knutzen, "Functional Anatomy of the Upper Extremity," in Biomechanical Basis of Human Movement, Second Edition, Second., Baltimore, MD: Lippincott Williams \& Wilkins, 2003, pp. 150-165.

[7] G. L. Soderberg, Kinesiology: Application to Pathological Motion. Baltimore, MD: Williams \& Wilkins, 1986.

[8] C. T. Wadsworth, "The Hand and Wrist," in Orthopedic and Sports Physical Therapy, J. A. Gould and G. J. Davies, Eds. St. Louis: Mosby, 1985, pp. 437-475.

[9] J. R. Doyle and M. J. Botte, Surgical Anatomy of the Hand \& Upper Extremity. Philadelphia: Lippincott Williams \& Wilkins, 2003. 
[10] E. Kertcher, "Activities of Daily Living," in The SAGE Encyclopedia of Intellectual and Developmental Disorders, E. B. Braaten, Ed. Thousand Oaks: SAGE Publications, Inc., 2018, pp. 19-22.

[11] P. Bobos, E. A. Lalone, R. Grewal, and J. C. MacDermid, "Do Impairments Predict Hand Dexterity After Distal Radius Fractures? A 6-Month Prospective Cohort Study," Hand, vol. 13, no. 4, pp. 441-447, 2018.

[12] T. R. Turgeon, J. C. MacDermid, and J. H. Roth, "Reliability of the NK dexterity board," J. Hand Ther., vol. 12, no. 1, pp. 7-15, 1999.

[13] M. Vergara, J. L. Sancho-Bru, V. Gracia-Ibáñez, and A. Pérez-González, “An introductory study of common grasps used by adults during performance of activities of daily living," J. Hand Ther., vol. 27, no. 3, pp. 225-234, 2014.

[14] T. G. Sevene, J. Berning, C. Harris, M. Climstein, K. J. Adams, and M. DeBeliso, "Hand Grip Strength and Gender: Allometric Normalization in Older Adults and Implications for the NIOSH Lifting Equation.," J. lifestyle Med., vol. 7, no. 2, pp. 6368, 2017.

[15] I. K. Haugen et al., "Prevalence, incidence and progression of hand osteoarthritis in the general population: the Framingham Osteoarthritis Study," Ann. Rheum. Dis., vol. 70, no. 9, pp. 1581-1586, 2011.

[16] D. J. Hunter, "Osteoarthritis," Best Pract. Res. Clin. Rheumatol., vol. 25, pp. 801814, 2011.

[17] J. Falconer, "Hand osteoarthritis," vol. 17, no. 2, pp. 47-50, 2012.

[18] C. F. Dillon, R. Hirsch, E. K. Rasch, and Q. Gu, "Symptomatic Hand Osteoarthritis in the United States," Am. J. Phys. Med. Rehabil., vol. 86, no. 1, pp. 12-21, 2007. 
[19] Y. Zhang, J. Niu, M. Kelly-Hayes, C. E. Chaisson, P. Aliabadi, and D. T. Felson, "Prevalence of symptomatic hand osteoarthritis and its impact on functional status among the elderly: The framingham study," Am. J. Epidemiol., vol. 156, no. 11, pp. 1021-1027, 2002.

[20] P. Bobos, G. Nazari, M. Szekeres, E. A. Lalone, L. Ferreira, and J. C. MacDermid, "The effectiveness of joint-protection programs on pain, hand function, and grip strength levels in patients with hand arthritis: A systematic review and metaanalysis," J. Hand Ther., 2018.

[21] F. Schick et al., "A biomechanical model of the wrist joint for patient-specific model guided surgical therapy: Part 2," Proc. Inst. Mech. Eng. Part H J. Eng. Med., vol. 230, no. 4, pp. 326-334, 2016.

[22] J. L. Sancho-Bru, “A 3D Biomechanical Model of the Hand for Power Grip,” J. Biomech. Eng., vol. 125, no. 1, p. 78, 2003.

[23] N. K. Fowler and A. C. Nicol, "A force transducer to measure individual Finger loads during activities of daily living," J. Biomech., vol. 32, pp. 721-725, 1999.

[24] N. K. Fowler and A. C. Nicol, "Measurement of external three-dimensional interphalangeal loads applied during activities of daily living."

[25] K. N. An, E. Y. Chao, W. P. Cooney, and R. L. Linscheid, "Forces in the Normal and Abnormal Hand," J. Orthop. Res., vol. 3, pp. 202-211, 1985.

[26] E. Y. S. Chao, K. N. An, W. P. Cooney, and R. L. Linscheid, "Normative model of human hand," in Biomechanics of the Hand: A Basic Research Study, E. Y. S. Chao, Ed. Singapore: World Scientific, 1989.

[27] B. Weightman and A. A. Amis, "Finger joint force predictions related to design of joint replacements," J. Biomed. Eng., vol. 4, pp. 197-205, 1982.

[28] J. C. Yeo, C. Lee, Z. Wang, and C. T. Lim, "Tactile sensorized glove for force and motion sensing," in 2016 IEEE SENSORS, 2016, pp. 1-3. 
[29] P. C. Hsiao, S. Y. Yang, B. S. Lin, I. J. Lee, and W. Chou, "Data glove embedded with 9-axis IMU and force sensing sensors for evaluation of hand function," Proc. Annu. Int. Conf. IEEE Eng. Med. Biol. Soc. EMBS, vol. 2015-Novem, pp. 4631-4634, 2015.

[30] P. S. Helliwell, A. Hower, and V. Wright, "An evaluation of dynamic qualities of isometric grip strength.," Ann. Rheum. Dis., vol. 47, pp. 934-939, 1988.

[31] F. C. Su, H. Y. Chiu, J. H. Chang, C. F. Lin, R. F. Hong, and L. C. Kuo, "Jar-opening challenges. Part 1: An apparatus for assessing hand and finger torques and forces in a jar-opening activity," Proc. Inst. Mech. Eng. Part H J. Eng. Med., vol. 223, no. 1, pp. 121-129, 2009.

[32] E. K. J. Chadwick and A. C. Nicol, "A novel force transducer for the measurement of grip force," J. Biomech., vol. 34, pp. 125-128, 2001.

[33] A. A. Amis, "Variation of finger forces in maximal isometric grasp tests on a range of cylinder diameters," J. Biomech., vol. 9, pp. 313-319, 1987.

[34] M. Bouzit, G. Burdea, G. Popescu, and R. Boian, "The Rutgers Master II - New design force-feedback glove," IEEE/ASME Trans. Mechatronics, vol. 7, no. 2, pp. 256-263, 2002.

[35] N. Lu, C. Lu, S. Yang, and J. Rogers, "Highly sensitive skin-mountable strain gauges based entirely on elastomers," Adv. Funct. Mater., vol. 22, no. 19, pp. 4044-4050, 2012.

[36] A. L. Window, Strain Gauge Technology. London: Elsevier Applied Science, 1992.

[37] J. Cordey and E. Gautier, "Strain gauges used in the mechanical testing of bones Part II: 'In vitro' and 'in vivo' technique,' Inj. Int. J. Care Inj., vol. 30, no. 1, pp. 14-20, 1999. 
[38] A. L. Solevåg et al., "Quantifying force application to a newborn manikin during simulated cardiopulmonary resuscitation," J. Matern. Neonatal Med., vol. 29, no. 11, pp. 1770-1772, 2016.

[39] L. K. Baxter, Capacitive Sensors: Design and Applications. New York: IEEE Press, 1997.

[40] C. Boustedt, U. Nordenskiöld, and Å. Lundgren Nilsson, "Effects of a hand-joint protection programme with an addition of splinting and exercise," Clin. Rheumatol., vol. 28, no. 7, pp. 793-799, 2009.

[41] W. Zhang et al., "EULAR evidence based recommendations for the management of hand osteoarthritis: Report of a Task Force of the EULAR Standing Committee for International Clinical Studies Including Therapeutics (ESCISIT)," Ann. Rheum. Dis., vol. 66, no. 3, pp. 377-388, 2007.

[42] P. A. Dieppe and L. S. Lohmander, "Pathogenesis and management of pain in osteoarthritis," Lancet, vol. 365, no. 9463, pp. 965-973, 2005.

[43] S. Dahaghin, S. M. A. Bierma-Zeinstra, A. Z. Ginai, H. A. P. Pols, J. M. W. Hazes, and B. W. Koes, "Prevalence and pattern of radiographic hand osteoarthritis and association with pain and disability (the Rotterdam study).," Ann. Rheum. Dis., vol. 64, no. 5, pp. 682-7, 2005.

[44] N. Smaby, M. E. Johanson, B. Baker, D. E. Kenney, W. M. Murray, and V. R. Hentz, "Identification of key pinch forces required to complete functional tasks," vol. 41, no. 2, pp. 215-223, 2004.

[45] G. Kulothungan, M. Nachiappan, M. S. Kumar, and S. Rajasekaran, "Comparative Study of Forces Involved in Different Styles of Handwriting," J. Autom. Control Eng., vol. 1, no. 3, pp. 260-264, 2013.

[46] A. Skiadopoulos and T. Lango, "Analysis of the Pressure Exerted by the Surgeon's Hand and Fingers Using a Novel Robotic Laparoscopic Instrument During Urethrovesical Anastomosis," Society of American Gastrointestinal and Endoscopic 
Surgeons. 2016.

[47] R. Sasikumar and K. Lenin, "Assessing the influence of hand-arm posture on mechanical responses of the human hand during drilling operation," Int. J. Adv. Manuf. Technol., vol. 93, no. 1-4, pp. 375-384, 2017.

[48] J. C. MacDermid and M. Mule, "Concurrent validity of the NK hand dexterity test," vol. 6, no. 2, pp. 83-93, 2001.

[49] J. C. MacDermid, "Development of a scale for patient rating of wrist pain and disability," J. Hand Ther., vol. 9, no. 2, pp. 178-183, 1996.

[50] C. Gummesson, I. Atroshi, and C. Ekdahl, "The disabilities of the arm, shoulder and hand (DASH) outcome questionnaire: Longitudinal construct validity and measuring self-rated health change after surgery," BMC Musculoskelet. Disord., vol. 4, pp. 1-6, 2003.

[51] P. J. Klompenhouwer, C. Lysack, M. Dijkers, and A. Hammond, "The Joint Protection Behavior Assessment: A Reliability Study," Am. J. Occupatioonal Ther., vol. 54, no. 5, pp. 516-524, 2000.

[52] S. Werle et al., "Age- and Gender-Specific Normative Data of Grip and Pinch Strength in a Healthy," Hand, The, pp. 76-84, 2009.

[53] L. M. Connelly, "Bland-Altman Plots,” vol. 17, no. 3, pp. 175-176, 2008.

[54] R. L. Chatburn and C. Clinic, "Evaluation of instrument error and method agreement," AANA J., vol. 64, no. 3, pp. 261-268, 1996.

[55] Q. Fu and M. Santello, "Improving fine control of grasping force during hand-object interactions for a soft synergy-inspired myoelectric prosthetic hand," Front. Neurorobot., vol. 11, no. JAN, pp. 1-15, 2018.

[56] S. B. Godfrey, M. Bianchi, A. Bicchi, and M. Santello, "Influence of force feedback on grasp force modulation in prosthetic applications: A preliminary study," Proc. 
Annu. Int. Conf. IEEE Eng. Med. Biol. Soc. EMBS, vol. 2016-Octob, pp. 5439-5442, 2016.

[57] B. A. Ekelman et al., "Occupational Therapy Interventions for Adults with Rheumatoid Arthritis: An Appraisal of the Evidence," Occup. Ther. Heal. Care, vol. 28, no. 4, 2014.

[58] A. Hammond, "Joint Protection: enabling change in musculoskeletal conditions," Int. Handb. Occup. Ther. Interv. Second Ed., pp. 607-608, 2015.

[59] K. Sakuma et al., "Wearable Nail Deformation Sensing for Behavioral and Biomechanical Monitoring and Human-Computer Interaction," Sci. Rep., vol. 8, no. 1, pp. 1-11, 2018. 


\section{Curriculum Vitae \\ Michael L. Riddle}

\section{EDUCATION}

Ph.D. Student in Biomedical Engineering
The University of Western Ontario (London, ON)
School of Biomedical Engineering
Thesis: "Four-Dimensional Computed Tomography to Examine
Dynamic Wrist Motion"
Supervisor: Dr. Emily Lalone
M.E.Sc. Candidate in Biomedical Engineering
The University of Western Ontario (London, ON)
School of Biomedical Engineering
Thesis: "Evaluation of Finger Forces During Activities of Daily $-2019 \quad \begin{aligned} & \text { Supervisors: Dr. Emily Lalone and Dr. Louis Ferreira } \\ & \text { Status: Thesis Defense Only }\end{aligned}$
B.S. Biomedical \& Mechanical Engineering (Double Major)
Rose-Hulman Institute of Technology (Terre Haute, IN)
Areas of Concentration: Biomechanics and Biomedical
Instrumentation

\section{PUBLICATIONS}

1. Riddle, M, Robinson, S., MacDermid, J., Szekeres, M., Ferreira, L., Lalone, E. "Evaluation of Individual Finger Forces During Activities of Daily Living In Healthy Participants and those with Hand Arthritis." Journal of Hand Therapy: Innovation Special Issue. Invited Submission: May 2019.

2. Gray, R., Thom M., Riddle, M., Suh, N., Burkhart, T., Lalone, E. "Image Based Comparison between the Bilateral Symmetry of the Distal Radii through Established Measures." Journal of Hand Surgery. Submitted: May 2, 2018.

3. Riddle, M., MacDermid, J., Holland, S., Lalone, E., Ferreira, L. "Wearable Strain Gauge Based Technology Measures Manual Tactile Forces during Activities of Daily Living." Journal of Rehabilitation and Assistive Technologies Engineering (Special Collection: Wearable Technologies for Active Living and Rehabilitation). Accepted: July 4, 2018. 


\section{ABSTRACTS/PRESENTATIONS AT CONFERENCES}

1. Riddle M., Robinson S., MacDermid J., Szekeres M., Ferreira L., Lalone E., "Determination of Individual Finger Forces During Activities of Daily Living." London Health Research Day, London, ON (April 30, 2019)

2. Riddle M., Suh N., Lalone E., Lee T., "Dynamic F-DCFPyL Imaging in Patients Following Wrist Fracture." Imaging Network Ontario, London, ON (March 28-29, 2019); Poster

3. Riddle M., Ronata P., Gray R., Suh N., Lee T., Lalone E., "4DCT to Examine Wrist Contact Mechanics." Imaging Network Ontario, London, ON (March 28-29, 2019); Poster

4. Riddle M., Robinson S., MacDermid J., Szekeres M., Ferreira L., Lalone E., "Evaluation of Individual Finger Forces During Activities of Daily Living." Ontario Biomechanics Conference, Alliston, ON (March 8-10, 2019); Poster

5. Riddle M., MacDermid J., Lalone E., Ferreira L., "Wearable Technology Measures Forces in the Hand During Activities of Daily Living." American Society of Biomechanics Conference, Rochester, MN (August 8-11, 2018); Poster

6. Riddle M., MacDermid J., Lalone E., Ferreira L., "Wearable Technology Measures Forces in the hand During Activities of Daily Living." Canadian Orthopedic Research Society Annual Meeting, Victoria, BC (June 20-23, 2018); Poster

7. Riddle M., MacDermid J., Lalone E., Ferreira L., "A Novel Method of Evaluating Finger Forces During Activities of Daily Living." Canadian Bone and Joint Conference, London, ON (May 8-10, 2018); Poster

8. Riddle M., MacDermid J., Lalone E., Ferreira L., "Wearable Technology to Measure Forces in the Hand During Activities of Daily Living." London Health Research Day, London, ON (May 8, 2018); Poster

9. Riddle M., MacDermid J., Lalone E., Ferreira L., "Technology-Enabled Joint Protection Program to Enhance Hand Function in Patients with Arthritis." Orthopedic Research Society, New Orleans, LA (March 10-13, 2018); Poster

10. Gray R., Thom M., Riddle M., Suh N., Burkhart T., Lalone E., “Image-Based Comparison Between the Bilateral Symmetry of the Distal Radii through Established Measures." Orthopedic Research Society, New Orleans, LA (March 10-13, 2018); Poster

11. Riddle M., MacDermid J., Lalone E., Ferreira L., "Technology-Enabled Joint Protection Program to Enhance Hand Function in Patients with Arthritis." The Arthritis Society Tour, London, ON (October, 2017); Poster 


\section{TEACHING EXPERIENCE}

\section{The University of Western Ontario, London, ON}

May - Aug. 2019 Teaching Assistant, School of Biomedical Engineering

Course Instructor: Dr. Emily Lalone

Course Title: Fundamentals of Biomedical Engineering Design

Jan. - April 2019 Teaching Assistant, Dept. of Mechanical and Materials Engineering Course Instructor: Dr. Hamidreza Abdolvand

Course title: Properties of Materials

- Led weekly tutorials to review course material

- Wrote questions for and marked weekly quizzes

- Proctored weekly quizzes, midterms and final exam

Sept. - Dec. 2018 Teaching Assistant, Dept. of Mechanical and Materials Engineering Course Instructor: Nikolas Knowles

Course title: Properties of Materials

- Led weekly tutorials to review course material

- Proctored weekly quizzes, midterms and final exam

- Marked quizzes

August 2018

Instructor, Western Engineering Summer Academy Course Title: Introduction to Biomechanics

- Course instructor on Anatomy, Biomechanics of the Hand, Diseases of Joints, and Treating/Repairing Diseased Joints.

Jan. - April 2018 Teaching Assistant, Dept. of Electrical \& Computer Engineering Course Instructor: Dr. Raveendra Rao

Course title: Electric Circuits and Electromechanics

- Held regular office hours to handle student questions

- Led course laboratory assignments

- Graded course assignments and lab reports

Sept. - Dec. 2017 Teaching Assistant, Dept. of Chemical \& Biochemical Engineering Course Instructor: Dr. Jin Zhang Course title: Fundamentals of Biochemical/Environmental Eng.

- Organized tutorial discussions over relevant course material

- Held regular office hours to handle student questions

- Proctored midterm and final exams 
Rose-Hulman Institute of Technology, Terre Haute, IN, USA

Feb. - May 2017 Teaching Assistant, Department of Mechanical Engineering Course Instructor: Dr. Glen Livesay

Course Title: Statics and Mechanics of Materials

Jan. - Feb. 2017 Teaching Assistant, Dept. of Biology and Biomedical Engineering Course Instructor: Dr. Bill Weiner

Course Title: Intro to Biomedical Engineering Lab Practices

Sept. - Nov. 2016 Teaching Assistant, Department of Mechanical Engineering Course Instructor: Dr. Alan Chiu

Course Title: Electrical Systems

2012-Present

Private/Community Tutor

Tutoring students from grade 5 - university in Mathematics, Science, and Engineering 